\title{
Meta
}

Journal des traducteurs

Translators' Journal

\section{Figement et lexicographie bilingue : contraintes linguistiques, pragmatiques et stratégies d'appropriation}

\section{Gérard Petit et Evangélia Liberopoulou}

Volume 53, numéro 2, juin 2008

La traduction des séquences figées

The Translation of Frozen Sequences

URI : https://id.erudit.org/iderudit/018519ar

DOI : https://doi.org/10.7202/018519ar

Aller au sommaire du numéro

Éditeur(s)

Les Presses de l'Université de Montréal

ISSN

0026-0452 (imprimé)

1492-1421 (numérique)

Découvrir la revue

Citer cet article

Petit, G. \& Liberopoulou, E. (2008). Figement et lexicographie bilingue : contraintes linguistiques, pragmatiques et stratégies d'appropriation. Meta, 53(2), 269-293. https://doi.org/10.7202/018519ar
Résumé de l'article

La lexicographie bilingue grec moderne-français/français-grec moderne reflète la position paradoxale de chaque langue : si l'apprentissage du grec moderne en France ne répond à aucun impératif, en revanche le français est, en Grèce, la seconde langue vivante obligatoire dès le secondaire. Les dictionnaires bilingues sont massivement rédigés et édités en Grèce, et destinés à des apprenants grecs. La question se pose alors de savoir dans quelle mesure leur conception résulte effectivement de l'observation des données lexicales des langues en présence, et si cette observation n'est pas doublée, filtrée par une pré-appropriation qui apprêterait le matériau à la langue cible pour en faciliter l'apprentissage. Le présent travail vise à ouvrir la réflexion sur les stratégies d'appropriation linguistiques et culturelles à l'oeuvre dans la lexicographie bilingue. Une de ces stratégies consiste à conformer le matériau d'entrée à certaines caractéristiques structurelles de L2. Notre axe d'observation portera sur la dichotomie entre construction synaptique et construction morphologique, qui permet d'opposer les deux langues sur un axe macrostructurel.
Ce document est protégé par la loi sur le droit d'auteur. L'utilisation des services d’Érudit (y compris la reproduction) est assujettie à sa politique d'utilisation que vous pouvez consulter en ligne.

https://apropos.erudit.org/fr/usagers/politique-dutilisation/ 


\title{
Figement et lexicographie bilingue: contraintes linguistiques, pragmatiques et stratégies d'appropriation
}

\author{
GÉRARD PETIT \\ Université Paris $X$, Paris, France \\ gerarpetit@wanadoo.fr
}

EVANGÉLIA LIBEROPOULOU

CEA

\begin{abstract}
RÉSUMÉ
La lexicographie bilingue grec moderne-français/français-grec moderne reflète la position paradoxale de chaque langue: si l'apprentissage du grec moderne en France ne répond à aucun impératif, en revanche le français est, en Grèce, la seconde langue vivante obligatoire dès le secondaire. Les dictionnaires bilingues sont massivement rédigés et édités en Grèce, et destinés à des apprenants grecs. La question se pose alors de savoir dans quelle mesure leur conception résulte effectivement de l'observation des données lexicales des langues en présence, et si cette observation n'est pas doublée, filtrée par une pré-appropriation qui apprêterait le matériau à la langue cible pour en faciliter l'apprentissage. Le présent travail vise à ouvrir la réflexion sur les stratégies d'appropriation linguistiques et culturelles à l'œuvre dans la lexicographie bilingue. Une de ces stratégies consiste à conformer le matériau d'entrée à certaines caractéristiques structurelles de L2. Notre axe d'observation portera sur la dichotomie entre construction synaptique et construction morphologique, qui permet d'opposer les deux langues sur un axe macrostructurel.
\end{abstract}

\section{ABSTRACT}

Bilingual Modern Greek/French - French/Modern Greek lexicography is a reflexion of the paradoxical situation of the two languages involved. Whereas there is no pressing need in France to learn Modern Greek, in Greece, French is the second obligatory foreign language taught from secondary school on. There are thus many bilingual dictionaries written and published, aimed at the market of Greek learners. The question arises as to the extent to which these dictionaries have been modelled on the actual observation of the lexical data of the languages involved and whether there is not some pre-appropriation bias seeking to fit the data to the target language in order to facilitate the learning process. This article aims at shedding light on the strategies involved in acquiring language and culture deployed in bilingual lexicography. One of these strategies is to mould the original linguistic data into certain structures which are characteristic of the second language. The focus here is on the dichotomy between synaptic and morphological constructions, inviting a comparison of the two languages from a macrostructural viewpoint.

\section{MOTS-CLÉS/KEYWORDS}

appropriation, figement, lexicographie bilingue, pragmatique, unité lexicale 


\section{Introduction}

Il n'est pas abusif d'affirmer que les dictionnaires constituent, entre autres, des répertoires d'enregistrement et de traitement du figement lexical. Dans ce contexte, les dictionnaires bilingues sont confrontés à une difficulté supplémentaire: ils ont pour fonction d'assurer la traduction en L2 (langue cible) d'une séquence figée (désormais SF) de L1 (langue source) et non pas (simplement) l'explicitation de son sens par un jeu de traduction intralinguale ${ }^{1}$. Cette activité se dédouble pour répondre à deux contraintes, constitutives de l'activité lexicographique bilingue. L'une est linguistique: il s'agit de proposer en L2 un équivalent d'une séquence linguistique de L1, aussi proche que possible lexicalement et sémantiquement. La seconde est culturelle: l'équivalent doit faire sens dans la culture cible et ne pas se contenter de transposer le sens de la séquence linguistique de L1.

Dans l'usage, les dictionnaires bilingues appartiennent à la bibliographie d'apprentissage des langues, même si leur conception ne les y prédispose pas toujours (Petit 2004). La lexicographie bilingue grec moderne-français/français-grec moderne reflète le statut paradoxal de chacune des langues au sein de la culture des deux pays. L'apprentissage du grec moderne, en France, n'est associé à aucun enjeu économique ou culturel ${ }^{2}$. D'où son caractère relativement confidentiel ${ }^{3}$, l'essentiel du champ étant occupé par le grec ancien. À l'opposé, le français est, en Grèce, la seconde langue vivante obligatoire dès le secondaire, après l'anglais. Son enseignement est actif, même s'il a connu un recul ces dernières années. Le français en Grèce dispose d'une saillance qui fait défaut à son homologue sur l'Hexagone. Spécificité éditoriale, les dictionnaires bilingues précités sont rédigés et édités en Grèce, et destinés massivement à des apprenants grecs. La question se pose alors de savoir dans quelle mesure leur conception résulte effectivement de l'observation des données lexicales du français. Réciproquement, il est permis de se demander si cette observation n'est pas doublée, filtrée par une pré-appropriation qui apprêterait le matériau à la langue cible afin d'en faciliter l'apprentissage.

D’une manière plus générale, le présent travail vise à ouvrir la réflexion sur les stratégies d'appropriation linguistiques et culturelles à l'œuvre dans la lexicographie bilingue. En règle générale, les études portant sur la traduction ou la lexicographie bilingue sont orientées vers l'aval: de la langue source vers la langue cible, les questions relatives à la traduction obéissant à la même vectorialisation. Nous aimerions dans le présent travail inverser la perspective et remonter de l'aval vers l'amont en observant comment celui-ci est conditionné par l'autre. Nous formons l'hypothèse qu'une de ces stratégies consiste à conformer le matériau d'entrée (les unités lexicales [désormais UL] de L1 recensées dans la nomenclature et comme matériau défini dans la microstructure) à certaines caractéristiques structurelles de L2. Notre axe d'observation portera sur la dichotomie entre construction synaptique et construction morphologique, qui permet d'opposer les deux langues sur un axe macrostructurel. Les SF constituent un matériau privilégié pour l'identification des stratégies dans la mesure où leur opacité et leur résistance à la traduction rendent ces stratégies visibles à l'analyse. Notre corpus sera constitué de deux dictionnaires français-grec: Néo

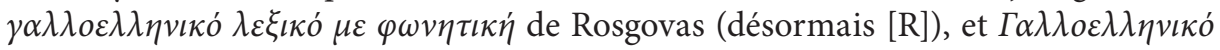

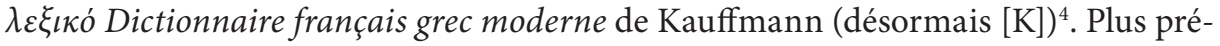
cisément, elle portera sur la lettre C, qui regroupe approximativement $10 \%$ des 
entrées et sous-entrées ${ }^{5}$. La première partie sera consacrée à l'étude des particularités fonctionnelles du grec et du français au regard de la construction d'unités complexes. Dans une seconde partie, nous envisagerons la négociation de ces spécificités par les dictionnaires, les présupposés qui la sous-tendent, sa finalité et la perspective induite en sortie sur le figement lexical du français (hexagonal).

\section{Figement et UL}

Comme tout ouvrage dédié à la description du lexique, les dictionnaires, qu'ils soient monolingues ou bilingues, sont confrontés à la délicate question du recensement des SF et de leur traitement. Celle-ci se double d'une difficulté complémentaire, liée à la variation dans la conception de l'unité lexicale ${ }^{6}$, selon qu'elle est pensée par la linguistique, la lexicographie ou la conscience courante des locuteurs. Toute perspective sur le figement convoquant nécessairement, à un moment ou l'autre, une réflexion sur l'unité lexicale, ici se séparent ce que l'on pourrait appeler des savoirs savants (élaborés notamment par la linguistique), des savoirs naïfs ${ }^{7}$ et la culture lexicographique.

\section{a. Deux divergences fonctionnelles entre langues}

Les savoirs savants relèvent d'une conceptualisation lexicologique dans le meilleur des cas, ou du moins sémantique ${ }^{8}$. Le champ se caractérise de longue date par une activité intense dont témoigne la profusion de propositions formulées en la matière (notamment Fradin 2003, Cadiot et alii 1997a, b, c, d, e, Cadiot et Lebas 2003, Franckel et alii 1996?, Franckel et Lebaud 1990, Camus et de Voguë 2004, Rey 1970, 1976), et qui animent la réflexion sur l'UL depuis quelques décennies. Toutefois, dans leur grande majorité, ces modèles se calent sur le mot graphique comme format emblématique de l'UL. Par là même ils se privent d'une ouverture et influent, en sortie, sur la configuration même de l'objet dont ils cherchent à décrire les caractéristiques. Les travaux sur le figement (Gross 1988, 19969., Mejri 1997, 2000, 2004. , Mejri, Baccouche, Clas et Gross 2003, Mejri, Baccouche, Clas et Gross 2000a et b) ont pour leur part l'intérêt (et le mérite) de recentrer la réflexion sur le caractère matriciel du signe, qui de ce fait n'est pas considéré comme un donné, mais comme une unité à construire. Dans ce cadre, est considérée comme UL toute séquence présentant un degré d'arbitrarité ${ }^{10}$ entre forme de l'expression et forme du contenu (selon une terminologie hjelmslévienne), lequel se traduit de manière différenciée selon les séquences. De ce fait, le statut d'UL ${ }^{11}$ recouvre des séquences dont le format n'est pas prédéterminé, mais s'échelonne sur un continu allant du mot graphique à la locution, en passant par les mots composés, collocations et les prédicats nominaux à verbes supports ${ }^{12}$. Ainsi, seront tenues pour UL des unités comme:

- chien, chat, maison, c'est-à-dire des UL monolexicales totalement arbitrarisées. S’y ajoutent des UL dont la signification n'est pas analysable, p. ex. gendarme, mais qui peuvent présenter des traces de motivation susceptibles à leur tour de donner naissance à des remotivations sauvages (choucroute);

- anthropologie, neurologue, neurosciences, raisonnable réalisent une configuration différente. Le format de leur signifiant est toujours celui du mot graphique, mais leur interprétation intègre une composante intrinsèquement motivée $e^{13}$, justifiant la scission 
de leur signifié en sens prédictible et sens lexical (Corbin 1987, Corbin et Temple 1994; Temple 1996). Ces UL constituent le centre prototypique du lexique construit;

- cache-col, cul-de-sac illustrent un cas analogue, mais caractérisé par une dissociation graphique du signifiant. C'est au niveau de cette classe d'unités que commence à se poser crucialement la question de l'identité lexicale ${ }^{14}$. L'absence de soudure plaide pour une construction syntaxique. Pourtant l'existence d'un trait d'union, constituant à part entière de l'orthographe des mots, vient rappeler que l'unité en question s'inscrit dans une zone hybride entre le lexique et la syntaxe. L'argument du trait d'union est mis à profit par la Morphologie lexicale pour capter ce genre d'unité et l'intégrer dans le lexique construit à l'aide de règles proprement morphologiques;

- pomme de terre, nez aquilin, boîte aux lettres sont des synapsies. L'absence de trait d'union, mais surtout la présence d'un marqueur syntaxique dans les réalisations $\mathrm{N} / \mathrm{N}$ les classe usuellement dans le lexique construit par la syntaxe ${ }^{15}$. Nous avons montré dans Petit (1998) que leur interprétation mobilise les mêmes ressorts et obéit à des contraintes analogues à celles des items construits par l'application de règles morphologiques;

- avoir faim, prendre peur sont respectivement représentatifs des prédicats nominaux à verbe support (Gross 1996) et des verbes figés. Ces unités sont considérées comme pleinement construites par la syntaxe. Toutefois elles héritent d'une structure sémantique dédoublée analogue à celle des mots morphologiquement construits. Elles s'en éloignent toutefois du fait de la généralité intensionnelle du verbe recteur (ici avoir, prendre...), laquelle a pu être parfois assimilée à une forme sémantiquement vide. La compositionnalité dont témoigne leur interprétation marque que le pivot notionnel de leur structure sémantique est localisé sur le nom. Ces séquences constituent par ailleurs, avec les locutions (cf. plus bas), le domaine de prédilection des études sur le figement;

- manger les pissenlits par la racine est une locution figée. L'inventaire de ce type de matériau est très vaste et les propriétés, tant formelles que sémantiques, complexes à décrire (Gross 1996, Mejri 1997, Grossmann et Tutin 2002, Petit 1998, 2003). Les locutions constituent des macrosignes, pouvant avoir la structure et le format de syntagmes complets. Elles constituent la part de la phraséologie qui échappe à la syntaxe. Leur intégration dans le lexique fait néanmoins l'objet de réticences dans la mesure où elles appartiennent à une zone périphérique qui échappe traditionnellement à la perception de l'UL. Ces séquences sont parfois analysables. Elles présentent en règle générale une résistance plus ou moins grande aux transformations que tolèrent habituellement les syntagmes libres. Par ailleurs, elles sont dotées d'une valeur dénominative (Mejri 1997, 2000). Les locutions constituent l'ultime maillon phraséologique du figement lexical ${ }^{16}$.

Ce bref et schématique panorama n'a pour objet que de montrer la diversité dimensionnelle des séquences susceptibles d'être captées par la notion d'UL. La question se pose toutefois de savoir si elle reflète une réalité translinguistique ou une spécificité du français relativement au grec moderne. L'observation des données discursives et lexicographiques permet d'établir une tendance forte de cette langue, qui ne mobilise pas les mêmes ressources que le français pour la construction de ses unités lexicales. D’une manière générale, le grec moderne se singularise par le fait qu'il a recours plus volontiers que le français à la construction morphologique. Ainsi, si les UL monolexicales et non construites sont effectivement attestées: $\gamma \alpha \dot{\alpha} \alpha$ (chat), $\mu o \lambda v^{\prime} \beta$ (crayon), $\kappa \alpha \lambda o ́ \varsigma$ (bon), en revanche une inflexion notable privilégie le recours au lexème monolexical pour l'expression du complexe, quel que soit la nature de son équivalent en français. De fait, on peut noter les correspondances suivantes (nous rappelons: $\mathrm{L} 1=$ français; L2 = grec moderne) ${ }^{17}$ : 


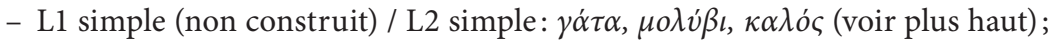

- L1 simple / L2 construit morphologiquement:

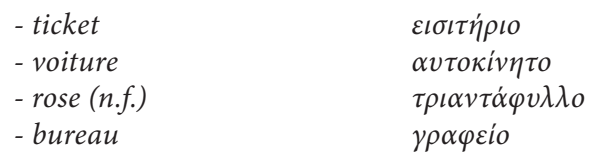

- L1 construit morphologiquement / L2 simple: pomme de terre / $\pi \alpha \tau \alpha \dot{\tau} \tau \alpha^{18}$;

- L1 construit morphologiquement / L2 construit morphologiquement:

$\begin{array}{ll}\text { - vitesse } & \tau \alpha \chi v i \tau \eta \tau \alpha \\ \text { - personnel (n.) } & \pi \rho o \sigma \omega \pi \iota \kappa o ́ \\ \text { - linguistique } & \gamma \lambda \omega \sigma \sigma o \lambda o \text { jí }^{2}\end{array}$

- L1 construit morphologiquement / L2 construit syntaxiquement: frites / $\pi \alpha \tau \alpha \dot{\tau} \tau \varepsilon \varsigma$ $\tau \eta \gamma \alpha v \imath \tau \dot{\varepsilon} \varsigma$

- L1 construit syntaxiquement / L2 construit morphologiquement:

- chemise de nuit

- nettoyage à sec

- cabinet médical

$v v \chi \tau \iota \kappa \iota \alpha ́$

- chargé d'affaires

- château d'eau

- avoir de la place

- avoir faim

- chambre à coucher

- grec moderne

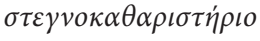

yı $\leftarrow \rho \varepsilon i o$

$\varepsilon \pi \iota \tau \varepsilon \tau \rho \alpha \mu \mu \varepsilon \dot{\varepsilon} \omega \circ$

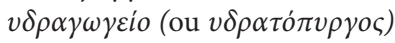

$\chi \omega \rho \alpha \dot{\omega} \omega$

$\pi \varepsilon \iota v \alpha \dot{\alpha} \omega$,

$\kappa \rho \varepsilon \beta \alpha \tau o \kappa \alpha \dot{\alpha} \mu \alpha \rho \alpha$

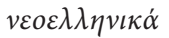

- L1 construit syntaxiquement / L2 construit syntaxiquement

- carte de crédit

- chef de rayon

- chauffage central

$$
\begin{aligned}
& \pi \iota \sigma \tau \omega \tau \iota \kappa \dot{~} \kappa \dot{\alpha} \rho \tau \alpha \\
& \pi \rho 0 \ddot{\sigma} \sigma \alpha \mu \varepsilon v o \varsigma \tau \mu \eta \dot{\mu} \mu \alpha \tau \sigma \varsigma
\end{aligned}
$$

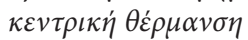

Il convient également de citer les inévitables idiosyncrasies lexicales dans le découpage du réel, qui entravent tout jeu de correspondance entre langues: $\beta$ ov $\eta^{\prime} \mu \alpha \tau \alpha$ ([tout viennoiserie, biscuit] que l'on consomme en trempant dans du café, du thé),

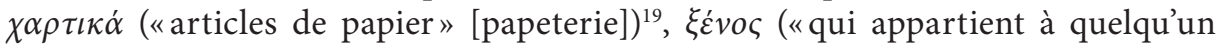
d'autre»), pour n'en prendre que quelques unes.

Une difficulté posée par le grec moderne réside dans le statut lexical incertain d'un nombre important de formations synaptiques. Si des expressions comme:

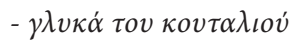

gâteaux à la cuillère ${ }^{20}$

- $\varepsilon \pi i \pi \lambda \alpha \kappa o v \zeta \iota v \alpha \varsigma$

- $\varepsilon \pi i \pi \lambda \alpha \mu \pi \alpha v \imath o v$

- $\varepsilon i \delta \eta \delta \omega \rho \omega v$

meubles de cuisine

meubles de salle de bain

cadeau $x^{21}$

sont effectivement intégrées à la compétence des locuteurs car attestées, il reste à déterminer si elles appartiennent pour autant au lexique de la langue et à quel degré 22 . D'autres présentent une structure syntaxique NN, AdjN qui les prédispose à la lexicalisation. Toutefois, l'instanciation lexicale de celle-ci incite à la prudence sur leur capacité dénominative: 


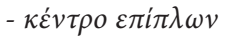

- $\sigma \kappa \lambda \eta \rho \dot{~} \varphi \dot{\varepsilon} \tau \alpha$

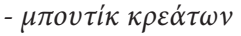

magasin de meubles ${ }^{23}$

feta dure ${ }^{24}$

boucherie ${ }^{25}$

Le partage dans la construction des noms et des verbes entre formation par application de règles et de formants morphologiques d'une part, et d'autre part par figement syntaxique semble bien avoir valeur signalétique en grec moderne. Le recours préférentiel au procédé morphologique est peut-être partie intégrante d'un ensemble d'habitudes inhérentes à la génétique des UL dans cette langue. Il n’en demeure pas moins que le mode de formation des mots complexes reflète leur propension à se lexicaliser. Si l'accès reste plus visible pour la formation morphologique, le figement constitue, par ses caractéristiques formelles, une zone d'extériorité au lexique. D'abord, par sa capacité à fournir des termes techniques, domaine où il reste bien représenté. Ensuite, du fait qu'il présente une propension à véhiculer des concepts au statut indéfini: (i) soit que ceux-ci présentent les caractéristiques nécessaires à l'identification de catégories référentielles, mais disposent d'un statut linguistique infra-

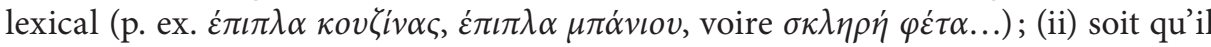
permette l'étiquetage de réalités déjà dénommées ailleurs, mais pour lesquelles il

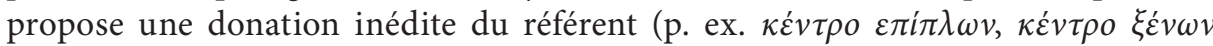

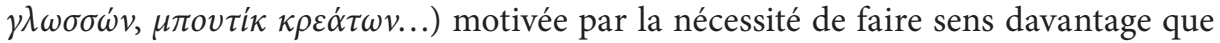
de catégoriser ${ }^{26}$. L'usage fait du figement réserve ce procédé à la constitution d'unités plus fragiles à la lexicalisation que la composition morphologique. Une telle répartition ne s'appuie sur aucun fondement linguistique à même de la justifier. Son principe semble bien être essentiellement culturel.

\section{b. Traitement lexicographique ordinaire du figement}

Cette configuration divergente entre modes de construction de dénominations complexe pose désormais la question de la gestion des divers modes de production lexicale par la lexicographie bilingue. En principe et par principe, la question ne doit pas interférer dans la sélection des données; la lexicalisation de la séquence fournit le critère de son enregistrement. Outre que ce principe souffre fréquemment d'exceptions, qui conduisent les lexicographes à supprimer des entrées nouvelles, à chaque édition d'un même ouvrage, c'est la conception même de l'unité lexicale qui est en jeu dans la représentation lexicographique.

Les dictionnaires généraux, qu'ils soient monolingues ou bilingues, observent au regard de l'unité de traitement la même attitude, qui génère deux contraintes sur le matériau à retenir. Celui-ci doit être lexicalisé (ou du moins supposé tel); il doit respecter le format du mot-forme. Si le premier critère témoigne d'une approche tendanciellement linguistique ${ }^{27}$, le second vient en limiter drastiquement la portée. Comme nous l'avons remarqué au paragraphe précédent, la notion d'UL n'impose pas de formatage a priori de la séquence. Toute contrainte sur ce plan résulte d'un paramétrage dont les tenants ne sont pas linguistiques mais reflètent des influences diverses: logiques, philosophiques, psychocognitives, éditoriales ${ }^{28}$, constitutives de la pratique lexicographique (bien que certaines d'entre elles interfèrent également dans une conception linguistique de l'UL). Sans oublier le poids d'une tradition qui remonte à la conception des premiers dictionnaires. 
La contrainte du mot-forme induit une perception hiérarchisée de la réalité lexicale: les séquences respectant la contrainte formelle précitée seront perçues comme prototypiques du lexique, les autres seront des unités périphériques, leur déclassement étant manifesté par la forme même de la construction, qui emprunte à la syntaxe. De fait, le lexique lexicographique se divise en deux catégories: des unités de premier rang, les mots-formes, auxquelles sera dévolue la position d'entrée d'article; des unités discontinues (synapsies, prédicats nominaux, locutions...) qui ne se verront traitées qu'à l'intérieur des articles.

Cette discrimination induit une perspective sur la signification lexicale. Le sens des unités figées sera supposé se résoudre à partir de celui du mot simple qui constitue la tête syntaxique de l'expression. Pour cette raison, les SF reçoivent un traitement qui soit les motive (et participe donc à des degrés divers à un défigement de leur représentation sémantique), soit les fige au risque de synthétiser abusivement leur structure sémantique selon qu'elles sont enregistrées à l'intérieur de la polysémie de l'article ou bien recensées dans une catégorie par défaut en fin d'article ${ }^{29}$.

Une difficulté supplémentaire vient brouiller davantage ce panorama, qui manque déjà singulièrement de clarté si ce n'est de logique: la conception que la lexicographie se fait du mot-forme ne coïncide pas avec celle, linguistique, du mot simple. Ainsi seront éligibles au titre d'entrées d'article (donc de représentants prototypiques du lexique) :

- des séquences monolexicales simples: chien, chat...;

- des séquences monolexicales construites par la morphologie: chorégraphie, chronomètre, centrifuge...;

- des séquences polylexicales dont les constituants sont séparés par un trait d'union: cardiovasculaire, céphalo-rachidien, couvre-lit...;

- des séquences polylexicales dont les constituants ne sont pas séparés par un trait d’union: curriculum vitae, caca d'oie, chemin de fer...

Si les deux premières catégories correspondent effectivement au format du $\operatorname{mot}^{30}$, en revanche la troisième ne s'y inscrit qu'en référence à une particularité typographique, la présence du trait d'union, qui vient abolir l'espace séparant les constituants, et par là même verser dans le monolexical, ce qui en fait ressortit de plein droit au polylexical, donc au figement. Toutefois, il est possible d'argumenter que la présence du trait d'union vient noter un stade avancé de lexicalisation, susceptible de laisser présager une soudure (cf. chèvrefeuille, contrebasse, croquemitaine) ${ }^{31}$. La quatrième catégorie constitue une dérogation à un principe institué pourtant par les lexicographes euxmêmes. Elle est imposée non pas par des considérations d'ordre morphologique mais par un faisceau diffus d'habitudes et de contraintes sémantiques. Statistiquement, son dénombrement est tout à fait résiduel. Chemin de fer en fournit un exemple. Aucun dictionnaire monolingue français ne traite ce $\mathrm{N}$ comme synapsie dans sa microstructure. De fait, son inscription à la nomenclature procède d'une naturalité sur laquelle aucun ouvrage français n'a jugé utile de revenir dans sa pratique. Elle est par ailleurs imposée par la structure sémantique de l'unité, qui n'admet pas une décomposition de son sens lexical (mais pas du sens prédictible, lequel produit le sens lexical par métaphore).

Les dictionnaires bilingues produits en Grèce ne s'affranchissent pas de cette procédure ${ }^{32}$. Ils la reflètent telle qu'elle est instanciée dans les ouvrages hexagonaux. 
Les unités monolexicales sont effectivement inscrites en entrées d'articles, suivant le même schéma que les dictionnaires français:

\begin{tabular}{|l|l|l|}
\hline & {$[\mathrm{K}]$} & {$[\mathrm{R}]$} \\
\hline - UL monolexicales simples & cône, concert, compte & chèque, chemise, cheveu \\
\hline - UL monolexicales complexes & $\begin{array}{l}\text { coagulation, coéquipier, } \\
\text { centrifugeuse }\end{array}$ & $\begin{array}{l}\text { catégoricité, cinémathèque, } \\
\text { cherté }\end{array}$ \\
\hline - UL polylexicales à trait d'union & $\begin{array}{l}\text { compte-chèque, chien-loup, } \\
\text { couvre-lit }\end{array}$ & $\begin{array}{l}\text { céphalo-rachidien, cupro- } \\
\text { alliage, contre-visite }\end{array}$ \\
\hline - UL polylexicales synaptiques & $\begin{array}{l}\text { caca d'oie, chemin de fer, } \\
\text { curriculum vitae }\end{array}$ & $\begin{array}{l}\text { cabin cruiser, chemin de fer, } \\
\text { curriculum vitae }\end{array}$ \\
\hline
\end{tabular}

Comme dans les dictionnaires monolingues français, cette dernière classe reste très pauvre quantitativement. Elle instancie un noyau (dur) de termes dont l'effectif restreint ne permet pas de caractériser un ouvrage relativement à l'autre. L'inscription de SF en entrées d'articles relève d'une posture paradoxale. D'abord, du fait qu'elle conduit l'ouvrage à déroger à l'un des principes fondamentaux de la pratique lexicographique. Ensuite, parce qu'elle se fonde massivement sur des unités que leur caractère stéréotypique rend incontournables en cette position ${ }^{33}$. Enfin, et subséquemment, parce qu'elle n'est pas l'occasion d'un investissement de la part du lexicographe, au même titre que la construction de la nomenclature monolexicale et polylexicale à trait d'union, mais d'une forme de retrait, de concession obligée.

La microstructure accueille pour sa part les SF, qu'elles appartiennent p. ex. à la classe:

\begin{tabular}{|l|l|l|}
\hline & {$[\mathrm{K}]$} & {$[\mathrm{R}]$} \\
\hline - des N composés synaptiques & $\begin{array}{l}\text { du carbonate de sodium, un } \\
\text { centre culturel, une réaction } \\
\text { en chaîne }\end{array}$ & $\begin{array}{l}\text { chef de rayon, central } \\
\text { téléphonique, pois chiche, } \\
\text { canne à sucre }\end{array}$ \\
\hline $\begin{array}{l}\text { - des verbes figés et des prédicats } \\
\text { nominaux à verbes supports }\end{array}$ & $\begin{array}{l}\text { faire une chute, avoir peur, } \\
\text { avoir faim }\end{array}$ & $\begin{array}{l}\text { avoir chaud, faire du charme, } \\
\text { prendre conscience de }\end{array}$ \\
\hline - des locutions & $\begin{array}{l}\text { parler de choses et d'autres, } \\
\text { etre porté sur la chose, } \\
\text { couper les cheveux en } \\
\text { quatre, faire son chemin }\end{array}$ & $\begin{array}{l}\text { se serrer la ceinture, avoir la de poule, être à cheval } \\
\text { sur, avoir le compas dans } \\
\text { l'œil }\end{array}$ \\
\hline
\end{tabular}

Dans les articles de [R], qu'ils soient ou non monosémiques, les SF apparaissent à la suite de la traduction du lexème entrée

\begin{tabular}{|c|c|}
\hline 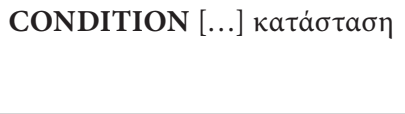 & $\begin{array}{l}\text { être en bonne }- \text { physique }[\ldots] / / / \text { conditions sociales, } \\
\text { politiques }[\ldots] / / \text { dans ces conditions }[\ldots] / / \text { à }- \text { de }[\ldots] / / \\
\text { à }- \text { que }[\ldots]\end{array}$ \\
\hline
\end{tabular}

\begin{tabular}{|c|c|}
\hline 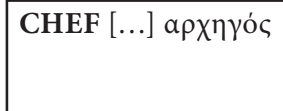 & $\begin{array}{l}\text { - de bord }[\ldots] / / \text { - de char } / /- \text { cuisinier }[\ldots] / / \text { - de gare } \\
{[\ldots] / /- \text { d'orchestre }[\ldots] / /- \text { d'accusation }[\ldots] / / \text { de son }} \\
\text { propre- }[\ldots] / /- \text { de rayon }[\ldots]\end{array}$ \\
\hline
\end{tabular}

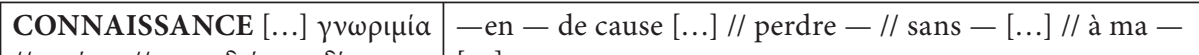

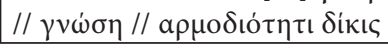
$[\ldots]$ 
sans qu'une logique de classement soit toujours perceptible ${ }^{34}$. Ce défaut n'est pas imputable à l'ouvrage en particulier, mais inhérent à la pratique lexicographique bilingue, dans la mesure où il se retrouve à l'identique dans un grand nombre d'autres dictionnaires du même type.

Les articles de $[\mathrm{K}]$ se distinguent du précédent en ce que l'inscription des SF est indexée sur la polysémie et, plus largement, le dégroupement des sens:

\begin{tabular}{|c|c|}
\hline 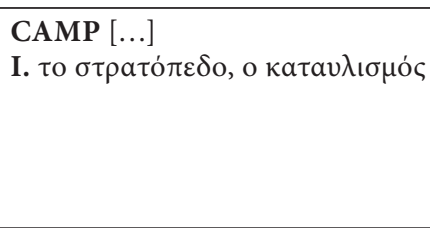 & $\begin{array}{l}\text { 1. un camp militaire }[\ldots] / / / \text { un lit de camp }[\ldots] \\
\text { 2. un camp de réfugiés }[\ldots] \text { // les camps de la mort }[\ldots] \\
\text { 3. changer de camp }[\ldots] \\
\text { 4. lever le camp }[\ldots] / / \text { ils ont fichu le camp }[\ldots] \text { // fous- } \\
\text { moi le camp }[\ldots]\end{array}$ \\
\hline II. $\eta \kappa \alpha \tau a \sigma \kappa \eta \dot{v} \nu \omega \sigma \eta$ & mon fils part en camp $[\ldots]$ \\
\hline
\end{tabular}

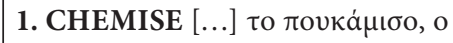

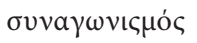

1. le col de la chemise [...]

2. la chemise de nuit $[\ldots]$

3. changer d'avis comme de chemise [...] // travailler en bras de chemise, en manches de chemise [...] // ils sont comme cul et chemise [...] // se soucier de quelque chose comme de sa dernière chemise [...] ranger des papiers dans une chemise [...]

Toutefois, à l'inverse de ce que l'on rencontre dans un dictionnaire monolingue, où elles suivent l'énoncé de la définition associée au sens concerné, elles fonctionnent par elles-mêmes comme indices de différenciation sémantique. Là non plus le procédé n'est pas spécifique au dictionnaire observé, mais plus généralement à la lexicographie bilingue. La négociation traditionnelle de cette fonction indicielle conduit fréquemment à un étagement de la polysémie: un premier niveau exprime le sens nucléaire à l'intérieur duquel des différentiations seront répertoriées et classées (par numéro d'ordre). Au second niveau se pose crucialement la question du figement, sous l'angle: (i) de son identification; (ii) de son expression; (iii) de son ordonnancement. Usuellement, le programme lexicographique bilingue impose que les significations du second niveau ne soient pas identifiées par une traduction mais pointées par une séquence discursive (exemple, SF...). De fait, la fonction de cette dernière devient ambiguë: noter une signification ou bien exprimer une SF. Aussi bien dans l'article camp que dans le double article chemise, il peut être délicat de distinguer avec certitude ce qui ressortit à l'indexation sémantique de ce qui procède du recensement des séquences figées. Ainsi, pour le premier, le statut de mon fils part en camp reste tout à fait incertain, tout comme à un autre niveau celui de ils ont fichu le camp et fous-moi le camp, qui sont pour leur part des SF non lemmatisées ${ }^{35}$. Dans l'article chemise, il est également permis de s'interroger sur le statut sémiotique de le col de la chemise.

Lordonnancement pour sa part résulte de choix qui restent implicites. Pour ne prendre qu'un exemple, la première acception de camp ( $\sigma \tau \alpha \tau$ có $\pi \varepsilon \delta o:$ "caserne», $\kappa \alpha \tau \alpha v \lambda \iota \sigma \mu o ́ \varsigma$ «campement») est axée sur la fonction ou la gestion militaire du référent, le fait que la population qu'il accueille y loge par contrainte ou nécessité, enfin que ces camps ont une fonction qui excède largement celle dévolue en principe à l'habitat. Les divisions sémantiques 3 et 4 ne sont impliquées dans cette acception que de manière tout à fait indirecte et mériteraient un traitement indépendant. 
La structure de l'article de dictionnaire bilingue présente un caractère paradoxal: d'une part, elle recense et explicite (en le traduisant, en le définissant ou en l'exemplifiant) du matériau linguistique mono et polylexical. D'autre part, s'appuyant sur un héritage qu'il ne lui appartient pas toujours de modifier ${ }^{36}$, elle crée les conditions d'un brouillage de l'information. Ces considérations d'ordre générique ne permettent toutefois pas d'expliciter un certain nombre de particularités inhérentes à la lexicographie français/grec moderne et à la gestion par les ouvrages de la divergence fonctionnelle existant entre les deux langues pour l'expression de données relevant, en français, du figement. C'est ce à quoi sera consacrée la seconde partie de l'étude.

\section{Figement et stratégies lexicographiques : quelques problèmes spécifiques aux dictionnaires du grec moderne}

Les dictionnaires bilingues français-grec moderne sont pris dans un double mouvement: (i) réinvestir dans leurs pratiques de description les habitudes précitées, héritées d'une tradition dont les tenants leur échappent; (ii) prendre en compte les contraintes pragmatiques qu'impose la situation lexicographique en contexte grec. Enfin, ils doivent intégrer, à des degrés divers, les particularités respectives des deux langues dans la construction des séquences complexes. Les ouvrages vont donc mettre en place des stratégies qui tendront soit à rapprocher la représentation qu'ils produisent du lexique de sa situation réelle en français, telle qu'elle peut être observée (auquel cas ils seront facilement manipulables pour un lecteur français), et de la perception qu'un locuteur français pourrait avoir de sa langue ${ }^{37}$, soit ils tendront à réduire l'effet d'écart entre L1 et L2 afin de produire une perception de familiarité entre les deux langues de façon à faciliter l'assimilation de $\mathrm{L} 1^{38}$. Les observations qui suivent porteront respectivement sur la nomenclature et la microstructure.

\section{a. La nomenclature comme reflet culturel de L2?}

$[\mathrm{K}]$ et $[\mathrm{R}]$ sont d'un volume analogue en nombre de signes. Toutefois, concernant le traitement des noms ${ }^{39}$, leur nomenclature présente des disparités de nature à les opposer. [K] enregistre 2459 noms à la lettre $\mathrm{C}$, contre 3440 pour [R], soit un dépassement de $28 \%$ pour ce dernier. Mais ce n'est pas tant la quantité en elle-même qui est révélatrice que sa ventilation.

La part des noms simples (non construits par la morphologie ou la syntaxe) est respectivement pour chaque ouvrage de 1277 entrées dans [K] contre 1361 dans [R]. Globalement, les dictionnaires enregistrent une proportion assez voisine de noms simples $([\mathrm{R}]$ n'excède $[\mathrm{K}]$ que de $6,17 \%)$. Ces mots représentent toutefois $28 \%$ de la nomenclature des noms pour $[R]$ contre $51 \%$ dans $[K]$. En clair, $[R]$ accorde une place plus importante aux noms complexes que son concurrent. La part réservée aux mots construits (par la morphologie et la syntaxe) s'établit comme suit: 1182 pour [K] contre 2079 pour [R], c'est-à-dire respectivement $48 \%$ et $60 \%$ de la nomenclature de la lettre C. Les deux dictionnaires adoptent donc des postures différentes au regard du lexique français: ils s'appuient sur un socle commun de mots simples, mais [R] augmente sa nomenclature en y injectant une proportion très importante de mots construits. Toutefois, pour en mesurer l'impact, encore faut-il la ventiler, elle aussi. C'est ce que propose le tableau ci-dessous: 


\begin{tabular}{|l|l|l|l|l|}
\hline Lettre C & {$[\mathbf{K}]$} & \% de la nomenclature & {$[\mathbf{R}]$} & \% de la nomenclature \\
\hline dérivés morphologiques & $\mathbf{9 9 5}$ & $40 \%$ & $\mathbf{1 4 1 5}$ & $41,13 \%$ \\
\hline composés morphologiques & $\mathbf{5 3}$ & $2,15 \%$ & $\mathbf{4 9 1}$ & $14,27 \%$ \\
\hline synapsies & $\mathbf{8}$ & $0,3 \%$ & $\mathbf{5}$ & $0,14 \%$ \\
\hline mots à trait d'union & $\mathbf{1 0 3}$ & $4,18 \%$ & $\mathbf{1 6 0}$ & $4,65 \%$ \\
\hline Total & $\mathbf{1 1 6 0}$ & $47,17 \%$ & $\mathbf{2 0 7 1}$ & $60,20 \%$ \\
\hline
\end{tabular}

Quelques remarques d'ordre quantitatif s'imposent avant de procéder à une analyse des types et formants lexicaux. La proportion de synapsies est aussi résiduelle dans $[R]$ que dans $[K]$, ce qui, compte tenu de l'ampleur de la nomenclature du premier, rend plus prégnant l'effet de dilution $(0,14 \%)$. En ceci les ouvrages ne font que reproduire un comportement largement attesté en lexicographie, bien que ne procédant d'aucune théorisation ou conceptualisation explicite du lexique. La proportion de mots à trait d'union est sensiblement analogue elle aussi ${ }^{40}$, mais peut être interprétée différemment. En principe, la construction de ces mots fait appel à des formants français, c'est-à-dire à des unités non issues du fonds savant. Dans ce cas, si l'on peut voir de la part de [K] une sorte d'adaptation de sa nomenclature à une habitude lexicographique observable, en revanche, il est permis de se demander dans quelle mesure la représentation proposée par $[R]$ ne procède pas d'une sous-pondération du fait même de la nature des formants à instancier. Ce questionnement peut trouver une validation si l'on prend en compte les proportions accordées aux autres modes de formation des mots complexes. La quantité de dérivés morphologiques est supérieure chez $[\mathrm{R}]$, mais reste, en données constantes, tout à fait analogue à celle de $[\mathrm{K}]$. En revanche, c'est du côté de la composition morphologique que l'écart va se creuser de manière significative: $3,09 \%$ chez $[\mathrm{K}]$ avec 53 entrées, contre $14,27 \%$ pour $[R]$, qui totalise 491 entrées, soit une progression de 89,20\%, largement supérieure à la différence quantitative entre les nomenclatures des deux ouvrages. Un constat s'impose: [R] s'oriente massivement sur la voie de la composition morphologique dans l'expression du complexe. Précisons notamment que ce mode de construction n'interdit pas, bien au contraire, l'emploi de formants appartenant au fonds savant. Toutefois, seule une étude appuyée sur les réalisations lexicosyntaxiques effectives permet de valider cette hypothèse.

$[\mathrm{K}]$ ne présente pas une vision homogène de sa conception des synapsies. Sa nomenclature intègre chemin de fer, caca d'oie, chasse (d'eau), cheval d'arçon, chevaux de bois, chou à la crème, curriculum vitae et compte(-)courant. Outre que la graphie du dernier demeure incertaine (ainsi que sa classification), le mode d'enregistrement de chasse d'eau privilégie l'option du mot simple sur celle de la synapsie. Caca d'oie n'est un nom que pour une partie de ses emplois. Seules tentatives téméraires, enregistrer chevaux de bois et chou à la crème ${ }^{41}$ en entrée d'article. À noter toutefois que $[\mathrm{K}]$ innove dans la mesure où les synapsies proposées en entrées sont régulièrement traitées dans la microstucture par les dictionnaires monolingues français (exception faite de chemin de fer et curriculum vitae). [R] pour sa part reste plus prudent mais peut-être moins attentif. À côté de chemin de fer et curriculum vitae, il enregistre cabin cruiser, casus belli et noix de coco (à coco). Si ce dernier peut valablement figurer dans un dictionnaire de niveau collège ou lycée, en revanche, il est permis de s'interroger sur les deux autres. Cette extériorité n'est pas tant une idiosyncrasie que la conséquence de choix ${ }^{42}$ dont les composés morphologiques présenteront une extension très 
large (voir plus bas). Toutefois, comme son concurrent, ce dictionnaire réserve le traitement du figement à la microstructure, donc considère les SF comme un matériau de seconde importance.

La gestion des mots à trait d'union est le lieu d'une inscription plus régulière, tant par $[\mathrm{R}]$ que par $[\mathrm{K}]$. Les deux ouvrages exploitent cette classe pour intégrer des paradigmes de composés de type N-(Prép.)-N ou V-(Prép.)-N. Ainsi, pour s’en tenir aux formules présentant plus de deux occurrences, on relève respectivement dans chacun des ouvrages:

\begin{tabular}{|c|c|c|c|c|}
\hline unité tête & occurrences & dans $[\mathrm{K}]$ & occurrences & dans $[R]$ \\
\hline cache-N & 6 & $\begin{array}{l}\text { cache-cache, cache- } \\
\text { col, cache-nez, cache- } \\
\text { pot, cache-radiateur, } \\
\text { cache-sexe }\end{array}$ & \begin{tabular}{|l|l}
7 \\
\end{tabular} & $\begin{array}{l}\text { cache-cache, cache-col, cache- } \\
\text { entrée, cache-flamme, cache-nez, } \\
\text { cache-pot, cache-sexe }\end{array}$ \\
\hline casse- $\mathrm{N}$ & 8 & $\begin{array}{l}\text { casse-cou, casse- } \\
\text { croûte, casse-gueule, } \\
\text { casse-noisette, casse- } \\
\text { noix, casse-pieds, } \\
\text { casse-pipe, casse-tête }\end{array}$ & 7 & $\begin{array}{l}\text { casse-cou, casse-croûte, casse- } \\
\text { noisette, casse-noix, casse-pieds, } \\
\text { casse-pierres, casse-tête }\end{array}$ \\
\hline chasse-N & 3 & $\begin{array}{l}\text { chasse (d'eau), chasse- } \\
\text { mouches, chasse-neige }\end{array}$ & 3 & $\begin{array}{l}\text { chasse-marée, chasse-mouches, } \\
\text { chasse-neige }\end{array}$ \\
\hline chauffe-N & 3 & $\begin{array}{l}\text { chauffe-biberon, } \\
\text { chauffe-eau, chauffe- } \\
\text { plats }\end{array}$ & 3 & $\begin{array}{l}\text { chauffe-bain, chauffe-biberon, } \\
\text { chauffe-eau }\end{array}$ \\
\hline compte-N & 5 & $\begin{array}{l}\text { compte-chèque, } \\
\text { compte-gouttes, } \\
\text { compte-rendu, } \\
\text { compte-tours, } \\
\text { compte(-)courant }\end{array}$ & 3 & $\begin{array}{l}\text { compte-gouttes, compte-rendu, } \\
\text { compte-tours }\end{array}$ \\
\hline contre-N & 13 & $\begin{array}{l}\text { contre-attaque, } \\
\text { contre-courant, } \\
\text { contre-espionnage, } \\
\text { contre-expertise, } \\
\text { contre-indication, } \\
\text { contre-indiqué, } \\
\text { contre-jour, contre- } \\
\text { offensive, contre-pied, } \\
\text { contre-plaqué, contre- } \\
\text { proposition, contre- } \\
\text { révolution, } \\
\text { contre-visite }\end{array}$ & 40 & $\begin{array}{l}\text { contre-amiral, contre-appel, } \\
\text { contre-assurance, contre-attaque, } \\
\text { contre-courbe, contre-courant, } \\
\text { contre-culture, contre-écrou, } \\
\text { contre-enquête, contre-épreuve, } \\
\text { contre-espionnage, contre- } \\
\text { exemple, contre-expertise, contre- } \\
\text { feu, contre-filet, } \\
\text { contre-indication, contre- } \\
\text { indiquer, contre-jour, contre- } \\
\text { lettre, contre-manifestant, } \\
\text { contre-manifestation, contre- } \\
\text { mesure, contre-offensive, contre- } \\
\text { pente, contre-pied, contre-plaqué, } \\
\text { contre-porte, contre-pouvoir, } \\
\text { contre-projet, contre-propagande, } \\
\text { contre-proposition, contre- } \\
\text { publicité, contre-révolution, } \\
\text { contre-révolutionnaire, contre- } \\
\text { taille, contre-terrorisme, contre- } \\
\text { torpilleur, contre-valeur, } \\
\text { contre-visite, contre-voie }\end{array}$ \\
\hline coupe-N & 4 & $\begin{array}{l}\text { coupe-circuit, coupe- } \\
\text { feu, coupe-gorge, } \\
\text { coupe-papier }\end{array}$ & 7 & $\begin{array}{l}\text { coupe-cigares, coupe-circuit, } \\
\text { coupe-jambon, coupe-légumes, } \\
\text { coupe-ongles, coupe-papier, } \\
\text { coupe-racines }\end{array}$ \\
\hline
\end{tabular}




\begin{tabular}{|l|l|l|l|l|}
\hline court-N & 4 & $\begin{array}{l}\text { court-bouillon, court- } \\
\text { circuit, court-circuiter, } \\
\text { court-métrage }\end{array}$ & 6 & $\begin{array}{l}\text { court-bouillon, court-circuit, } \\
\text { court-circuiter, court-courrier, } \\
\text { court-jointé, court-jus }\end{array}$ \\
\hline couvre-N & 4 & $\begin{array}{l}\text { couvre-chef, couvre- } \\
\text { feu, couvre-lit, couvre- } \\
\text { pieds }\end{array}$ & 3 & couvre-feu, couvre-lit, couvre-plat \\
\hline cardio & 1 & cardio-vasculaire & 3 & $\begin{array}{l}\text { cardio-pulmonaire, cardio-rénal, } \\
\text { cardio-vasculaire }\end{array}$ \\
\hline $\begin{array}{l}\text { cul-de-N } \\
\text { / GN }\end{array}$ & 2 & $\begin{array}{l}\text { cul-de-jatte, cul-de- } \\
\text { sac }\end{array}$ & 3 & $\begin{array}{l}\text { cul-de-basse-fosse, cul-de-jatte, } \\
\text { cul-de-sac }\end{array}$ \\
\hline cupro-N & 0 & & 3 & $\begin{array}{l}\text { cupro-alliage, cupro-aluminium, } \\
\text { cupro-ammoniaque }\end{array}$ \\
\hline
\end{tabular}

Deux remarques s'imposent. L'approche paradigmatique permet aux dictionnaires de concilier la nomenclature avec un des objectifs assignés traditionnellement à l'enseignement du lexique: procéder par listes de mots ${ }^{43}$. Le procédé leur permet également d'amplifier leur nomenclature à moindre coût ${ }^{44} \mathrm{et}$, d'une certaine manière, d'optimiser l'ergonomie de la consultation.

C'est toutefois dans le type de mots que se marquera une différence qualitative entre ouvrages. Si $[K]$ s'en tient à des UL d'usage courant, $[R]$, en revanche, comporte une proportion non négligeable d'unités à caractère terminologique, avec parfois des lacunes surprenantes sur le vocabulaire non spécialisé (p. ex. le traitement des mots en chasse-N, court-N ou coupe-N). La tendance se confirme également pour les $\mathrm{N}$ possédant une ou deux attestations: café-concert, café-théâtre, caille-lait, céphalorachidien, cérébro-spinal, chaland-citerne, crête-de-coq (sic), cypho-scoliose dans [R] pour n'en citer que quelques uns. $[R]$ se caractérise par une plus grande proportion d'unités à formants savants (cardio-N et cupro-N). Ce dernier fait doit être mis en relation avec la composition morphologique, qui est le lieu où les deux dictionnaires divergent et où l'empreinte d'une orientation culturelle se dessine.

La nomenclature des composés morphologiques est extrêmement révélatrice. Dans [K], sur 53 unités procédant de la composition morphologique, seules 6 résultent de l'association de bases qui ne sont pas d'origine grecque: cedex (acronyme), celluloïd, centripète, chèvrefeuille, contrebasse et croquemitaine. Les autres composés (et leurs dérivés) sont soit issus intégralement de bases d'origine grecque: chromosome, chrysalide, cinémascope, coléoptère, soit de structures dont un constituant est grec: centigramme, centimètre, cancérologie, cardiologue, cancérigène, soit enfin de structures dont les bases sont latines mais néanmoins interprétables par transposition dans le grec moderne (nous soulignons): calorifère, calorifuge, centrifuge, centrifugeuse $^{45}$. La traduction de ces unités du français vers le grec s'opère d'ailleurs elle aussi sur le principe du calque total (série a), ou partiel (série b) :

- série a:

\begin{tabular}{|c|c|}
\hline cacophonie & $\kappa \alpha \kappa o \varphi \omega v i \alpha$ \\
\hline calorifère & $\kappa \alpha \lambda o \rho \iota \varphi \varepsilon ́ \rho$ \\
\hline chimiothérapie & $\chi \eta \mu \varepsilon \iota \circ \theta \varepsilon \rho \alpha \pi \varepsilon i \alpha$ \\
\hline cholestérol & 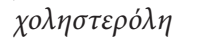 \\
\hline chronologie & 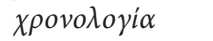 \\
\hline chlorhydrique & $v \delta \rho o \chi \lambda \omega \rho \iota k o ́ \varsigma$ \\
\hline coléoptère & 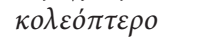 \\
\hline
\end{tabular}


- série b:

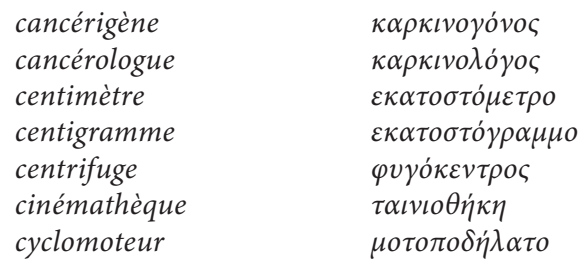

L'injection de ce type de mots dans la nomenclature réduit l'effet de distance entre les langues et tend à présenter l'une comme le reflet de l'autre. Dans le processus de transfert L1/L2, la question délicate est de savoir exactement comment s'établit ce reflet. Un mot français peut être construit sur des formants d'origine grecque tout comme un mot grec peut réactualiser les formants de sa langue au contact d'un mot français qui les lui a empruntés. Mais là n'est pas la question. La nomenclature apparaît comme le lieu d'une irruption possible de L2 à l'intérieur de L1 sous couvert de formants appartenant à L1. Sur le plan de l'ouvrage, la procédure de traduction s'en trouve affectée du fait que celle-ci se voit délestée d'une partie de son champ d'application au profit du recours à un jeu de biunivocité entre formants. La question se pose de la proportion à accorder en principe à ces calques. Ils sont inévitables, car ils reflètent une réalité linguistique et dont un dictionnaire bilingue doit rendre compte. Par ailleurs, la faible proportion de mots construits sur des bases savantes dans [K] ne permet pas de tirer de conclusion sur le caractère procédural de la démarche, à savoir si elle relève ou non d'une stratégie. Comme nous le verrons plus bas, la proportion de mots relevant du figement syntaxique vient compenser largement le phénomène.

La démarche adoptée par $[\mathrm{R}]$ diverge largement de celle de $[\mathrm{K}]$ par le fait que l'injection de construits savants est tout à fait massive (491 unités) et amplifiée par un effet de paradigmatisation dérivationnelle: $[\mathrm{R}]$ ne se contente pas d'enregistrer un construit morphologique, il produit dans toute la mesure du possible la liste (non exhaustive il est vrai) de ses dérivés:

- cancérologie, cancérologique, cancérologue

- catalyse, catalyser, catalyseur, catalytique

ou bien multiplie l'attestation de composés sur une même base N1:

- chronobiologie, chronogramme, chronographe, chronologie, chronologique, chronologiquement, chronométrage, chronomètre, chronométrique, chronophotographie

Ajoutons enfin qu'une partie de ces entrées appartient à une terminologie très spécialisée, dont la présence dans un dictionnaire général peut pour le moins surprendre:

- catabatique, castoréum, caryocinèse, carboxylhémoglobine, charadriiforme...

dans la mesure où elle semble peu compatible avec le profil non spécialiste requis pour le lecteur dans l'introduction. Il est donc permis de se demander si cette terminologie ne vient pas enfler artificiellement (et dans une proportion importante) la nomenclature au point de concourir, et même d'aboutir, à sa morphologisation. Comme nous le verrons au paragraphe suivant, le fait n'est pas compensé par une augmentation proportionnelle de la représentation du figement. Sachant que le grec moderne présente une forte propension à morphologiser la construction des noms 
complexes, il est permis de se demander dans quelle mesure la conception de la nomenclature n'est pas l'objet d'une procédure d'apprêt visant à refléter non pas tant la réalité lexicale du français que l'image qu'un locuteur grec véhicule inconsciemment de sa propre langue. En clair, la nomenclature française de [R] serait plus ou moins adaptée en fonction d'une règle de construction du grec moderne: L1 serait établie en fonction de la forme de L2. Le processus de traduction se voit d'autant gauchi qu'il est inversé: c'est en fonction de (la forme du construit de) L2 que va être recherché (le construit de) L1, étant entendu que le calque sémantique et morphologique fournira le principe régulateur de la sélection. Quelle est dans ce cas la part de la traduction? Elle mobilisera non pas des savoirs mais des jeux de correspondances biunivoques, (re)productibles par le lecteur sur la base d'une simple intuition de sa propre langue. Outre le fait que ceux-ci produiront chez lui le sentiment que le français est pour partie du grec réécrit (translittéré en fait), voire traduit, ils risquent de favoriser des erreurs sur des mots formellement apparentés mais dont l'interprétation s'appuie sur des ressorts différents.

\section{b. La microstructure et le figement}

Les deux dictionnaires enregistrent des séquences figées dans la microstructure, mais là aussi dans des proportions variables. La représentation des noms composés par la syntaxe (donc relevant du figement) est diversement pondérée selon les ouvrages. Si on la rapporte à la proportion des construits morphologiques, on obtient la répartition suivante:

\begin{tabular}{|l|l|l|l|l|l|}
\hline & {$[\mathbf{K}]$} & $\begin{array}{l}\text { \% de la } \\
\text { nomenclature }\end{array}$ & {$[\mathbf{R}]$} & $\begin{array}{l}\text { \% de la } \\
\text { nomenclature }\end{array}$ & $\begin{array}{l}\text { \% de }[\mathbf{R}] \\
\text { par rapp. à }[K]\end{array}$ \\
\hline composés morphologiques & $\mathbf{5 3}$ & $2,17 \%$ & $\mathbf{4 9 1}$ & $14,30 \%$ & $+926,40 \%$ \\
\hline composés syntaxiques & $\mathbf{5 8 2}$ & $23,90 \%$ & $\mathbf{3 6 8}$ & $10,72 \%$ & $-158 \%$ \\
\hline
\end{tabular}

Les deux dictionnaires respectent ici encore des logiques inverses. Si $[\mathrm{K}]$ reflète une tendance marquée du français à recourir à la synapsie pour la composition (Benveniste $1974 \mathrm{a}$ et b) nominale, surtout dans le domaine technique, [R] se caractérise par une représentation opposée, dans laquelle le construit syntaxique reste inférieur non seulement à la proportion d'unités enregistrées pour la même catégorie par $[\mathrm{K}]$, mais aussi relativement à celle des composés morphologiques intégrés en entrée par [R] lui-même. Le profil du lexique français proposé par ce dernier diverge non seulement de celui attesté chez son concurrent, mais surtout de ce que produirait une observation empirique minimalement informée. Rapportées à l'ensemble de la nomenclature des noms dans les deux ouvrages, les deux proportions confirment l'inversion de tendance. On peut fournir deux explications complémentaires à ce phénomène.

$[\mathrm{K}]$ n'opte pas pour un format particulier d'article, en termes de volume. Dans la très grande majorité des cas, il réserve sa microstructure à l'enregistrement de SF et de syntagmes discursifs pouvant être apparentés à des SF selon les cas. Ce matériau occupe effectivement une place prédominante dans la part laissée à la traduction de l'entrée. Un lissage des données, à considérer toutefois avec prudence, laisse apparaître une moyenne de 4 ou 5 séquences par article, quelle que soit la catégorie grammaticale à laquelle on puisse les rattacher ${ }^{46}$. Pour [R], les proportions sont différentes dans la mesure où le dictionnaire opte massivement pour un format bref (en moyenne 
3 lignes par article), dans lequel la polysémie éventuelle de l'entrée reste parfois délicate à identifier. Nous reproduisons ci-dessous les articles centimètre, central et cercle, qui peuvent être tenus pour représentatifs de la conception de ce dictionnaire, et des interrogations qu'elle peut susciter ${ }^{47}$ :

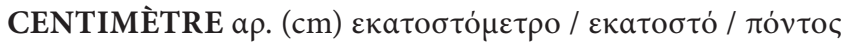

CENTRAL $\alpha \rho . \kappa \varepsilon \dot{v} \tau \rho o:-$ téléphonique

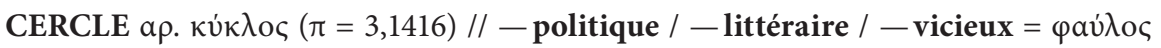

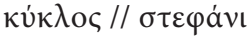

Du fait de la brièveté des articles, dont le modèle dominant est illustré ici par centimètre, le rapport entre segments discursifs (lesquels instancient une très grande proportion de SF) et articles tombe à 1 pour 4 articles, soit globalement l'inverse de la configuration attestée dans $[\mathrm{K}]$.

La seconde explication tient à l'enjeu associé au format bref. Celui-ci peut être interprété différemment. D’abord en termes d'accessibilité pour le public visé. Des articles courts sont plus aisément consultables par un lecteur peu expérimenté en L1. Toutefois, l'argument serait mis en défaut par l'orientation terminologique de la nomenclature. Autre hypothèse, vers laquelle nous penchons, un article bref, surtout dans le format adopté par [R] permet de réduire, si ce n'est d'éviter, l'instanciation de SF dans un projet lexical tourné essentiellement vers la morphologie. Autre avantage, très concret, la place gagnée par le volume réduit des articles permet d'augmenter le nombre de ceux-ci, donc d'amplifier l'injection d'unités morphologiquement construites. Représentation morphologique du lexique, sous-représentation du figement et réduction drastique du format des articles participent au même projet lexicographique: proposer un objet lexique en L1 qui soit le plus isomorphe possible à son correspondant de L2. Contrairement à celui de $[\mathrm{K}]$, le projet de $[\mathrm{R}]$ aboutit à la construction d'une fiction.

Il serait erroné toutefois de penser que l'opposition entre les deux ouvrages s'articule sur une dichotomie ne touchant que l'aspect quantitatif des données. Le mode d'enregistrement des séquences appelle des commentaires qui incitent à nuancer la perspective du fait qu'il implique une inversion de la perspective. Ici encore $[\mathrm{K}]$ et [R] s'opposent. Le matériau enregistré par le premier n'est pas systématiquement lemmatisé, mais libellé sous forme de syntagme dans l'immense majorité des cas. De fait, l'identification d'une SF devient fréquemment difficile à établir. Si :

- une crise cardiaque, le mètre cube, la critique littéraire, un critique littéraire, une culotte de cheval

relevés dans la microstructure, instancient effectivement des noms composés qui identifient chacun une catégorie référentielle, et sont facilement identifiables en tant que tels par tout locuteur francophone, en revanche:

- la racine cubique de 8 est 2

- faire le signe de croix

posent problème. En premier lieu est concerné le format de la séquence: dans la racine cubique de 8 est 2 , seul racine cubique est une SF, correspondant à un nom composé, la phrase globale ne fournissant qu'une contextualisation d'accueil pour son expression. Faire le signe de croix peut recevoir deux analyses: l'une réduirait la SF à la séquence signe de croix, qui correspond effectivement à un nom composé; une autre 
capterait la totalité de l'expression en s'appuyant sur le fait que faire constitue un opérateur approprié et que signe de croix présente les propriétés d'un prédicat nominal (si se signer est attesté en français, se signer de croix ou en croix ne le sont pas). Nous avons défendu cette dernière option dans Petit (1998).

Des réalisations comme:

- la croûte d'un fromage, le cul d'une bouteille, la cuisine électorale, une maladie curable, la cuvette des cabinets, les étudiants du premier cycle

n'accueillent pas, quant à elles, des SF. Les deux premières sont les doublons discursifs, c'est-à-dire sous forme de syntagmes libres, des noms composés: croûte de fromage ${ }^{48}$ et cul de bouteille. Cuisine électorale n'est pas un nom composé mais une collocation, c'est-à-dire une association privilégiée entre UL. Mais elle n'isole pas une catégorie référentielle, et ne peut donc prétendre en tant que telle à un statut lexical. En effet, cuisine dispose d'un emploi en français glosable par «manœuvre, comportement louche»; électorale est l'un des adjectifs qui accompagnent préférentiellement le nom, compte tenu du contexte situationnel auquel celui-ci s'applique. Maladie curable présente les mêmes caractéristiques que la précédente, à la différence près que l'adjectif admet d'occuper la position d'attribut, ce que reflète en grande partie ses contextes d'emploi. La collocation qu'il contribue à former avec maladie présente donc un degré de cohésion moindre que celui observable avec cuisine électorale ${ }^{49}$. Les deux dernières réalisations (la cuvette des cabinets, les étudiants du premier cycle) ne constituent pas en elles-mêmes des SF, les expansions droites étant régies uniquement par les lois de la grammaire. Le fait est inscrit dans leur structure syntaxique ${ }^{50}$.

Un autre fait est à prendre en considération: le regroupement ou le dégroupement des séquences au sein de la microstructure. Nous reproduisons ci-dessous le classement adopté par $[\mathrm{K}]$ des noms composés avec N1 = crème:

- la crème fraîche

- la crème chantilly

- la crème renversée, la crème caramel

- une crème au chocolat, à la vanille

- une crème antirides

- une crème de banane

- une crème de gruyère

- un café-crème

- un crème

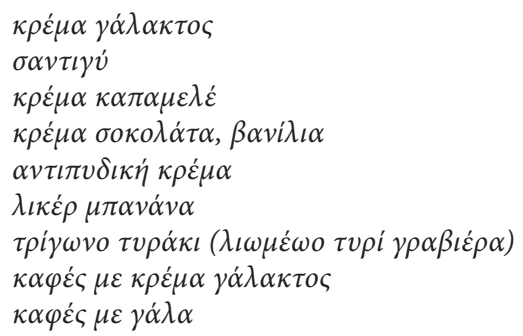

Il ne répond que partiellement aux contraintes de la traduction en L2, lesquelles auraient demandé à ce que les emplois régis par le même équivalent de traduction soient regroupés. En clair, ceux gouvernés directement ou non par $\kappa \rho \varepsilon ́ \mu \alpha$, et qui renvoient:

- à une substance [+ comestible] [- boisson];

- à une substance [- comestible] : $\alpha v \tau \iota \pi v \delta \iota \kappa \dot{~} \kappa \rho \varepsilon \dot{\mu} \mu \alpha$;

et ceux qui appartiennent au paradigme de $\lambda \iota \kappa \varepsilon ́ \rho:$ [+ comestible] [+ boisson].

Le dégroupement des emplois la crème renversée, la crème caramel, d'une part, et une crème au chocolat, à la vanille, de l'autre, ne correspond pas à une réalité linguistique de L2 ni de L1, tous se traduisant par $\kappa \rho \varepsilon ́ \mu \alpha$. Elle est justifiée par le souci de marquer la synonymie des deux premières expressions, oubliant au passage qu'une crème caramel peut également ressortir pleinement au type noté par crème au chocolat, 
c'est-à-dire impliquer une relation d'ingrédience au titre de laquelle le caramel interviendrait non pas comme garniture, mais comme composant intrinsèque de la crème. Cet emploi ne dispose pas, il est vrai, d'équivalent dénominatif en grec moderne. Il ressortit pourtant à la même catégorie que café-crème et crème (n.m.), qui eux non plus ne correspondent pas à des dénominations en L2 ${ }^{51}$. Les traductions qui leur sont appliquées sont des syntagmes explicatifs qui témoignent de la limite culturelle de juxtaposition entre systèmes lexicaux. Ce cas est illustré de manière plus nette par crème de gruyère, appellation générique inusitée aujourd'hui en France dans l'interlocution courante ainsi que dans les appellations employées par la (grande) distribution. La traduction proposée est une description en tout point analogue à celle que l'on rencontrerait dans un dictionnaire monolingue. À la différence de café-crème et crème (n.m.), crème de gruyère correspond davantage à une réalité alimentaire et culturelle répandue en Grèce ${ }^{52}$, le produit étant largement connu des locuteurs (notamment sous l'appellation commerciale de Vache qui rit).

D’une manière générale, $[\mathrm{K}]$ dissout les $\mathrm{SF}$ dans un moule rédactionnel inadapté, qui ne permet pas la discrimination entre le matériau lexicalisé et le syntagme libre. Par ailleurs, il semble bien que la conception du figement véhiculée par ce dictionnaire subordonne la nécessité de rendre compte des séquences polylexicales à la recherche d'un effet de réel: enregistrer des séquences de discours, quel que soit le type de matériau qu'elles véhiculent. C'est probablement pour cette raison que la conception du figement adoptée par $[\mathrm{K}]$ reste très aléatoire, au risque d'apparier des syntagmes libres à des traductions qui, elles aussi, ne s'appuient pas sur des SF.

[R] adopte une position différente. L'ensemble du matériau qu'il enregistre en microstructure est lemmatisé. De fait, tombe le reproche majeur que l'on pouvait faire à son concurrent d'instancier de manière indifférenciée et de ne pas donner à son lecteur les moyens de discriminer le matériau lexicalisé. Les articles central et cercle, cités plus haut, sont extraits de [R]. Ils adoptent une procédure qui s'appuie explicitement sur des règles en vigueur pour la citation du matériau lexical. Il en va de même pour:

CONGÉ ap. $(\mathrm{cm})$ á $\delta \varepsilon ı$ // - de maladie / - de maternité / - annuel // prendre a

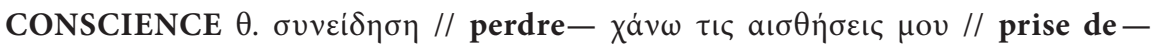

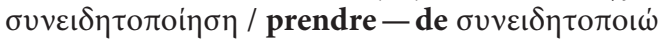

CORPS $\alpha \rho . \sigma \dot{\omega} \mu \alpha / /$ - d'homme / - médical /corps à corps $\sigma \dot{\omega} \mu \alpha \mu \varepsilon \sigma \dot{\omega} \mu \alpha / /$ - diplo-

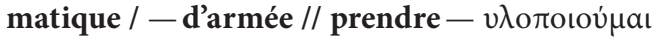

Le mode de traitement diffère toutefois de celui observé dans $[\mathrm{K}]$ dans la mesure où l'ouvrage tend à compacter un certain nombre de données de L1 et à les inscrire dans des suites énumératives dépourvues de traduction. S'il obéit à un impératif traductionnel, les équivalents en L2 étant les calques lexico-sémantiques des SF de L1, [R] laisse néanmoins induire que la structure compositionnelle de leur interprétation déborde le sens lexical ${ }^{53}$. Même s'il est vrai que congé de maladie / de maternité / annuel se traduisent par $\alpha \delta \varepsilon \iota \alpha+\mathrm{X}$, il n'en reste pas moins qu'une telle perspective occulte la forme de ces séquences en L2 (donc du figement), la structure lexico-syntaxique des traductions n'étant que partiellement donnée dans la langue cible ${ }^{54}$. De fait, le traitement apporté au matériau polylexical tend à hypothéquer la validité de la démarche entreprise sur le plan de la sélection et de la présentation de ces séquences. 
Autre particularité des occurrences retenues par [R], elles sont inspirées par les principes qui conduisent à l'établissement de la nomenclature de L1: un certain nombre de séquences polylexicales françaises se voient traduites en grec moderne par un équivalent morphologiquement construit ${ }^{55}$ :

\begin{tabular}{|c|c|}
\hline $\begin{array}{l}\text { - prendre congé } \\
\text { - prise de conscience } \\
\text { - prendre conscience de } \\
\text { - prendre corps }\end{array}$ & 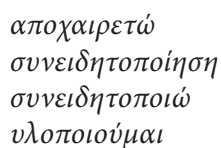 \\
\hline
\end{tabular}

au point qu'il est permis de se demander si l'ouvrage n'adopte pas là non plus une stratégie d'appropriation, définie depuis une connaissance plus ou moins conscientisée de L2. Cette tendance à subordonner l'équivalence sémantique à la propension de L2 à construire son matériau lexical par la morphologie se remarque dans d'autres articles, où le mouvement s'établit au détriment de l'isomorphisme entre langues ${ }^{56}$. Ainsi, aux articles main et mal, les SF du français sont elles massivement traduites en grec par des composés monolexicaux ${ }^{57}$ :

\begin{tabular}{|c|c|c|}
\hline main & $\begin{array}{l}\text { à-armée } \\
\text { sous la - } \\
\text { de longue - } \\
\text { - courante } \\
\text { faire - basse } \\
\text { prendre en - }\end{array}$ & 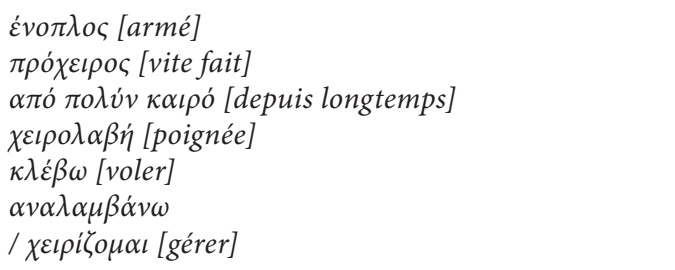 \\
\hline mal & $\begin{array}{l}\text { avoir - } \\
\text { - de dents } \\
\text { - de tête } \\
\text { avoir - aux dents } \\
\text { - de cour } \\
\text { avoir - au cour }\end{array}$ & 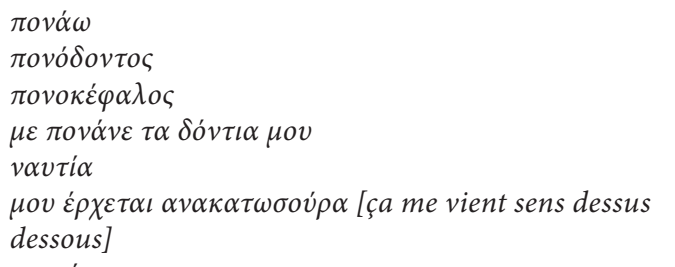 \\
\hline & $\begin{array}{l}\text { - de mer } \\
\text { avoirle - de mer }\end{array}$ & 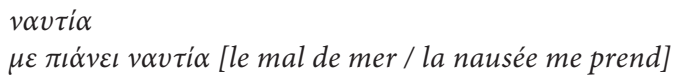 \\
\hline
\end{tabular}

\section{En guise de conclusion}

Il est prématuré de conclure tant le champ à explorer reste vaste. Les stratégies mises en place par les dictionnaires conjuguent deux arguments hétérogènes : respect de la forme lexicographique et perception des relations entre langues. Sur le premier plan, les ouvrages peuvent se fonder sur un modèle hérité d'une tradition qui prévoirait une nomenclature définie en fonction du profil de l'apprenant, ainsi que des articles regroupant le maximum de SF issues de L1. C'est cette perspective qu'adopte $[\mathrm{K}]$ et dont s'éloigne $[\mathrm{R}]$. Toutefois, pour être menée à bien, elle nécessite une perception claire $^{58}$ du statut lexical des séquences enregistrées. L’adoption d'une forme syntagmatique, si elle présente l'avantage de contextualiser les données, n’en demeure pas moins inadaptée à l'expression et au formatage des données lexicales. Par ailleurs, la perception par le lexicographe des relations structurelles entre langues peut être utile. Elle ne doit toutefois pas gauchir la représentation produite de L1. Pour cette raison, les dictionnaires produits par statistique d'occurrence (DAFA, DAFLES) présentent une garantie contre toute déviation de ce type. 
Les stratégies en place dans les dictionnaires bilingues français-grec reposent sur ce double mouvement de proximité ou d'éloignement de la sémiotique lexicographique par rapport à des règles ou du moins de grandes tendances observables entre langues opposées. La question de fond posée par ces stratégies est celle de la posture de l'ouvrage relativement au lecteur de L2, mais aussi de l'incidence de celle-ci sur la compréhension des données lexicales en L1. Opter pour la proximité (L1 établie en fonction des structures de L2) ne produit en sortie qu'un artefact de la langue source dont on voit mal quel pourrait en être l'usage pour un locuteur de L2, exception faite éventuellement dans le cadre d'une appropriation purement scolaire par un lecteur ${ }^{59}$ en contexte de version. L'extériorité pour sa part garantit un respect du profil formel de L1. Toutefois, elle n'est productive que si la métalangue de description se dote de moyens de contrôle efficaces de la polylexicalité.

Les dictionnaires, qu'ils soient monolingues ou bilingues, se construisent généralement par compilation, comme nous l'avons précisé. Un rapprochement de $[\mathrm{K}]$ et $[\mathrm{R}]$ montrerait que tel n'a pas été le cas ${ }^{60}$. Mais là n'est pas l'intérêt, ou le préjudice. La compilation produit un brassage des données qui peut se révéler fécond pour l'établissement d'un dictionnaire à venir. Dans le contexte de la lexicographie bilingue grec-français/français-grec, un ouvrage produit en France par des locuteurs français aurait permis un autre point de vue sur les deux langues, mais aussi l'établissement d'une autre sémiotique lexicographique. Les ouvrages élaborés dans les deux pays en auraient bénéficié. En définitive, ce dont souffrent les deux dictionnaires observés, mais qui n'est pas spécifique à eux, est l'unilatéralité de leur conception (dans tous les sens du terme). Ceci induirait-il que dans certains contextes l'élaboration d'un dictionnaire bilingue n'est réellement envisageable que s'il existe déjà un symétrique publié dans un pays disposant d'une forte tradition lexicographique? Il convient de rester prudent sur ce point, mais la question mérite d'être posée.

\section{NOTES}

1. Comme peut l'être l'explicitation du sens par une définition.

2. Contrairement au grec ancien.

3. Aucune structure pédagogique n'a été instituée par l'Éducation nationale française dans le secondaire. Il faut attendre le supérieur pour voir s'ouvrir, localement, des cursus destinés fréquemment aux grands débutants. Parallèlement existent des cours donnés par de petits instituts privés ou par l'Éducation nationale grecque aux enfants de la diaspora et à quelques curieux, en général dotés d'une solide motivation.

4. Ces deux ouvrages sont très largement diffusés en Grèce et connus du public. Le dictionnaire de Kaufmann a pour auteurs Colette Lust et Dimitri Pandelodimos. Leurs noms sont discrètement mentionnés à l'intérieur de l'ouvrage.

5. Quel que soit le dictionnaire concerné, bilingue ou non.

6. En règle générale, aucune approche du figement ne peut faire l'économie d'une réflexion sur l'unité lexicale dans la mesure où les deux données, bien qu'hétérogènes, sont structurellement et fonctionnellement liées (Mejri 1997).

7. Faute de place, nous ne les aborderons pas ici. Précisons néanmoins que la question est extrêmement complexe et qu'elle reste sous-estimée et sous-théorisée par la Linguistique. Citons pour repère le numéro 154 de la revue Langages, consacré aux représentations métalinguistiques ordinaires.

8. La morphologie lexicale reste, pour sa part, stérile à toute investigation heuristique sur l'unité lexicale, et ce, pour deux raisons. D'une part, à cause de sa conception de l'UL, massivement ancrée au mot graphique (Corbin 1987, Corbin et Temple 1994; Fradin 2003). Les questions relatives au figement lui sont soit invisibles, soit elles sont rejetées dans le champ de la syntaxe. Ici préside précisément la seconde cause de stérilité. La morphologie lexicale est actuellement prise dans un débat qui a pour objet de circonscrire pour elle un espace existentiel, et à même de lui assurer 
une légitimité dont elle se sent privée. Ceci la conduit, d'une part, à renégocier les relations entre flexion et dérivation. La morphologie constructionnelle entend par là se réapproprier un domaine qu'elle avait laissé en friche et montrer que les relations entre les deux facettes de la structuration du signifiant, loin d'être disjointes, sont complémentaires, chacune participant à la détermination de l'autre. Second point de débat, plus polémique (nous n'entrerons pas dans le débat) : les relations entre Morphologie et Syntaxe, notamment autour du mot composé. La morphologie utilise la notion de mot graphique comme emblème de la construction lexicale, renvoyant la synapsie à la Syntaxe. Cette opposition frontale, dans laquelle la Syntaxe fait figure d'agressée, n'a pas permis de déboucher sur une conception homogène et cohérente de l'UL.

9. Et, indirectement, Kleiber (1984) qui, bien que travaillant sur la dénomination, a mis l'accent sur la nécessité de prendre en compte le couplage séquence/référent pour l'identification des UL.

10. Décelable au fait que la décomposition d'un plan ne donne pas pleinement, et de manière biunivoque, accès à l'autre plan.

11. Nous nous limiterons ici aux UL caractérisant le lexique restreint ( $N, V$ ou Adj). Les propriétés du figement intéressent également les unités grammaticales, lesquelles à leur tour peuvent contenir des UL référentielles: à cause de, dans une proportion de, grâce à... Une analyse portant sur les deux types de séquences dépasserait toutefois le cadre de la présente étude.

12. Nous émettrons des réserves pour les phrases figées et les proverbes du fait qu'ils ne disposent pas d'une des caractéristiques essentielles de l'UL: s'intégrer syntaxiquement à un constituant supérieur. Si une intégration existe pour eux, elle n'est pas syntaxique mais textuelle.

13. Corollaire au fait que leur signifiant se laisse analyser.

14. Nous laissons de côté volontairement la question des séquences inférieures au mot graphique (bases autonomes ou non, affixes, allomorphes, fractomorphèmes, racines expressives) pour lesquelles se pose également la question de l'identité lexicale.

15. On peut rester dubitatif sur la validité d'une telle répartition dès que la séquence présente la structure $\mathrm{N}$ Adj. Comme le montrent les quelques exemples que nous proposons, une même structure, selon qu'elle présentera ou non le trait d'union, verra sa construction imputée à la morphologie ou à la syntaxe, indépendamment de sa structuration sémantique.

16. Exception faite, comme nous l'avons indiqué, des phrases figées, proverbes, dictons, dont l'intégration au lexique reste très discutable.

17. Le recensement proposé ici n’a qu'une valeur indicative et ne recouvre que des classes générales.

18. Cette configuration reste résiduelle. Elle est constituée essentiellement d'emprunts.

19. Les deux premiers mots sont des neutres pluriels et ont un sens de pluralité. Le dernier s'applique à n'importe quelle réalité dont la possession est attribuée à l'autre. Il serait illusoire et peu pertinent de dresser une liste de ces phénomènes et de leurs modes d'encodage. Ils sont particulièrement prégnants dans le domaine des traditions populaires (gastronomie notamment), mais aussi de l'enseignement des institutions juridiques et politiques, comme c'est le cas pour un grand nombre de langues (Szende [dir.] 2002). L'allusion que nous proposons n'a d'autre objet que d'attirer l'attention sur la mobilisation des procédés morphologiques.

20. Fruits, parfois légumes, confits au sirop.

21. Littéralement articles de cadeaux, objets à offrir, dénomination figurant sur les enseignes et vitrines.

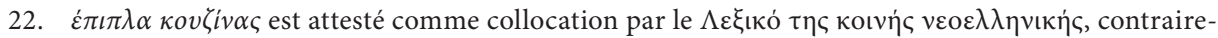
ment à $\dot{\varepsilon} \pi \imath \lambda \lambda \alpha \mu \pi \dot{\alpha} v ı o v$, qui ne fait l'objet d'aucune mention. Réciproquement, une consultation de supports lexicographiques et terminographiques démontrera le caractère précaire de meuble de cuisine et meuble de salle de bain en français. Ces expressions sont transparentes et présentent une disponibilité dénominative. Toutefois, elles ne sont pas tenues pour lexicalisées, et leurs équivalents mobilier de cuisine et mobilier de salle de bain disposent d'un statut lui aussi aléatoire. Mobilier de cuisine est absent du Grand dictionnaire terminologique et du Trésor de la langue française. Il figure comme collocation pour le Petit Robert. Réciproquement, meuble de cuisine n'est attesté que par le Trésor, qui l'envisage comme collocation. Meuble / mobilier de salle de bain n'est attesté dans aucun ouvrage. Ces deux expressions viennent suturer une lacune dans une architecture conceptuelle sans pour autant fournir de dénomination correspondante, si l'on en croit les dictionnaires. Une enquête sur un vaste corpus permettrait de trancher tant sur le plan de la forme que sur celui de la fonction sémiotique (une analyse linguistique démontrerait en revanche leur forte propension à occuper une fonction dénominative, et donc à intégrer le lexique).

23. Littéralement «centre de meubles». Le N $\kappa \varepsilon ́ v \tau \rho o$ ("centre») intervient fréquemment dans l'appellation de commerces et institutions à caractère culturel et/ou économique: $\pi v \varepsilon v \mu \alpha \tau \iota \kappa o ́ ~ \kappa \varepsilon ́ v \tau \rho o$ 


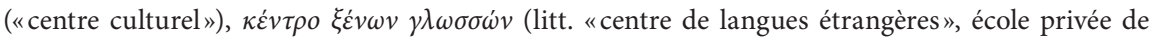
langues) sans que pour autant il aboutisse systématiquement à la constitution d'une dénomination.

24. L'expression figure sur l'étiquetage. Elle permet d'isoler une catégorie subordonnée, un type, sans que pour autant une garantie de lexicalisation soit apportée. La question est identique en français avec des expressions comme cantal jeune/vieux.

25. Littéralement «boutique de viandes». Cette dernière appellation, bien que formée de manière

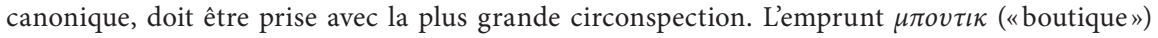
existe en grec moderne dans l'acception «magasin de vêtements». Bien qu'attestée sur quelques enseignes, la construction $\mu \pi \sigma v \tau \iota \kappa \kappa \rho \varepsilon \alpha \tau \omega v$ relève du néologisme si ce n'est de la désignation, au sens de Kleiber (1984).

26. Ou peut-être par la volonté de recatégoriser fictivement et symboliquement une réalité dont l'usage catégoriel veut être pointé comme usure.

27. Conception purement intériorisée, voire inconsciente. En règle générale, la lexicographie ne s'est jamais dotée d'une théorie explicite de l'UL et n'a jamais voulu emprunter aux modèles proposés par la Linguistique (le fait qu'ils se distinguent par leur hétérogénéité a largement contribué à ce rejet). Seuls les dictionnaires produits à l'appui de théories linguistiques - p. ex. Mel'čuk - se dotent d'une conception explicite et argumentée de l'UL. Toutefois ceux-ci constituent davantage des laboratoires d'analyse du sens et des propriétés lexicales que des ouvrages répondant aux exigences sociales des dictionnaires.

28. L'impact de ces dernières sur la configuration matérielle de l'ouvrage ne doit pas être sous-estimé. L'ergonomie de la consultation constitue une exigence à laquelle les lexicographes restent sensibles. Elle conditionne l'attitude du lecteur-client face à l'ouvrage et entre en compte dans la décision d'achat.

29. Traitées dans la polysémie, le sens de la tête est rapporté à celui de l'entrée; regroupées au sein d'une catégorie par défaut, la définition peut marquer abusivement la globalisation du sens. Ces types de traitements aboutissent à une impasse dans la mesure où la notion de degré de figement leur est invisible et qu'ils conduisent à une représentation uniforme de faits lexicaux qui devraient être distingués.

30. Raison pour laquelle, entre autres, la morphologie lexicale n'est qu'une morphologie du mot et n'a jamais cherché à développer de relations avec les études sur le figement. Mejri $(1997 ; 2000)$ et nous-même (Petit 1998; 2003) avons montré que des relations étroites existent entre les deux perspectives, tant concernant leur conceptualisation du signifiant que, essentiellement, celle de la structure sémantique des unités.

31. Les rectifications de l'orthographe (Journal officiel de la République française, année $1990 \mathrm{n}^{\circ} 100$, 6 décembre 1990) recommandent fortement aux lexicographes de procéder à la soudure quand aucune contrainte d'euphonie ne vient s'interposer.

32. Les dictionnaires, où qu'ils soient produits, sont massivement conçus par compilation. La nomenclature de $[\mathrm{R}]$ et $[\mathrm{K}]$ ne fait que révéler, dans ses principes, les modèles dont elle s'est inspirée. L'illusion serait de penser que ces nomenclatures ont été mises au point par les deux ouvrages, indépendamment de toute consultation de leurs homologues unilingues français.

33. Il est permis de se demander dans quelle mesure leur positionnement en entrée ne résulte pas davantage d'un comportement mimétique que de choix raisonnés.

34. Le critère de l'ordre alphabétique n'est que partiellement efficient comme le montrent les deux articles. Celui relatif à la position de l'unité au sein d'une séquence n'est guère plus probant.

35. Ce dictionnaire bilingue ne fait que reproduire une tendance largement attestée ailleurs, au point de devenir la règle du genre. Les dictionnaires de langue, qu'ils soient bilingues ou non, ne contrôlent qu'aléatoirement leur métalangue de description (et ce, malgré leurs dénégations). À notre connaissance, les seuls ouvrages à avoir fourni un effort significatif en ce sens pour le français sont le dictionnaire combinatoire de Mel'čuk et deux dictionnaires multilingues qui en sont inspirés, le DAFA et le DAFLES.

36. La question est très complexe. D’une part, le poids des habitudes dans la pratique lexicographique limite les interventions à des lissages de surface. Rares sont les ouvrages, pour le français, qui ont cherché à s'affranchir et à proposer une méthodologie indépendante (voir note précédente). Par ailleurs, il se trouve que ces pratiques héritées, si elles ont permis l'édification d'un ensemble d'habitudes devenues transparentes pour les concepteurs, ont conduit également à façonner des attentes chez les lecteurs. Ces derniers s'accommodent d'autant mieux des lacunes et déficits des ouvrages qu'ils les convertissent en expression d'un savoir-faire et d'une compétence dont ils se sentent dépourvus. 
37. Perception purement intuitive, née de la manière dont il a intégré la grammaire de sa langue et constituée de schémas plus ou moins précis sur son organisation.

38. Nous montrerons qu'en fait cet objectif n'est qu'un leurre, dont l'objet est de rassurer le lecteur en restreignant l'extériorité de la langue dite «étrangère».

39. Le figement est multiforme et intéresse plusieurs catégories grammaticales. Pour la présente étude nous nous limiterons aux noms.

40. Compte tenu du volume plus important (28\%) représentée par la nomenclature de [R], elle est, en données constantes, en légère progression chez ce dernier.

41. À noter toutefois que le procédé n'est pas généralisé puisque le patron $\mathrm{N}$ à la crème / au chocolat / à la sauce $\mathrm{N}$ reste improductif pour sa nomenclature. Il s'agit donc d'une inscription due uniquement au hasard.

42. Cabin cruiser, peu fréquent en français au point que l'on peut s'interroger sur sa représentativité, doit être perçu dans sa dimension d'anglicisme et ce, relativement à la culture des locuteurs grecs, pour lesquels l'étude de l'anglais débute parfois dès le primaire. Il n'est pas abusif de penser que l'inscription de ce terme peut avoir une valeur signalétique, tout comme l'aura celle des composés morphologiques.

43. En ceci les ouvrages font preuve de peu d'originalité relativement à l'évolution de la réflexion en la matière.

44. L'instillation de séries paradigmatiques représente un peu moins de la moitié des entrées à trait d'union pour $[\mathrm{K}]$ et presque $60 \%$ pour $[\mathrm{R}]$.

45. La base -fère peut être connectée à gr. $\varphi \varepsilon \varepsilon \omega \omega$ «porter» et -fuge à $\varphi \varepsilon v ́ \gamma \omega$ "partir». Toutefois, calorifère présente un cas particulier. Ce mot, construit sur le même schéma en français que conifère, ne se voit pas traduit en grec de la même manière que les composés en -fère. Si ces deux mots entretien-

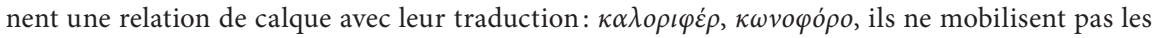
mêmes procédés. Le premier est un emprunt résultant de la transposition du signifiant phonique, tandis que le second entretient une relation de calque sémantique. Si, pour calorifère, le nom grec est une traduction à partir d'une L1, en revanche, il convient d'être prudent pour conifère (et plus généralement pour les noms en -fère).

46. Éventuellement aucune s'il s'agit de simples exemples maquillés en SF.

47. Citons entre autres la distribution aléatoire des SF (absence pour centimètre, une seule pour central). Le déficit de cohérence avec la traduction proposée pour l'entrée ( $\kappa \varepsilon \dot{\varepsilon} v \tau \rho o$ signifie également «centre»), et leur mode de traitement: (i) absence de traduction (le lecteur doit chercher les mots de la SF qu'il ignore à leur entrée; dénégation implicite du figement sémantique et de l'influence du contexte lexico-syntaxique pour la construction du sens lexical; à noter que $[\mathrm{K}]$ ne propose pas

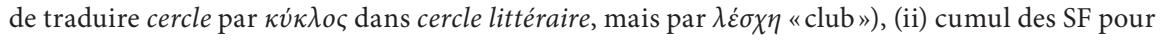
des valeurs différentes du $\mathrm{N}$ tête (la série gouvernée par cercle).

48. Dont le statut lexical peut être sujet à discussion.

49. Si l'on admet: cette maladie est curable, en revanche, on rejettera cette cuisine est électorale (électoral est un adjectif relationnel qui ne possède pas par ailleurs d'emploi qualificatif, contrairement à p. ex. parisien).

50. Même si les expansions permettent d'isoler des types. Toutefois, d'après nos recherches, cuvette de cabinet n'est pas attesté en tant que dénomination et étudiant de premier cycle reste d'un statut incertain.

51. Bien que $[\mathrm{K}]$ les distingue, le Petit Robert électronique considère ces deux expressions comme synonymes.

52. Nous resterons prudent sur l'implicite de cette affirmation dans la mesure où le statut catégoriel du café-crème en Grèce reste incertain face au café grec, au café français et au café frappé, qui se partagent le champ cognitif.

53. La proportion de séquences non lexicales reste résiduelle, à l'image de la faible proportion de séquences enregistrées en microstructure (corps d'homme).

54. On pourrait également s'étonner du classement des séquences. Ainsi, il est peu cohérent de séparer corps médical de corps diplomatique, etc.

55. La représentativité de ce type de configuration se voit renforcée localement par l'absence de traduction de séquences correspondant à une synapsie en L2 (corps d'homme / médical / diplomatique / d'armée vs prendre corps) ou par l'effet amplificateur produit par la déclinaison du paradigme morphologique des UL en L1 (prise de conscience / prendre conscience vs perdre conscience).

56. Encore doit-on préciser le domaine d'application de cette lacune. Il est vrai que les séquences proposées en L1 ne disposent pas nécessairement d'équivalent en L2 et présentent une structure 
sémantique rigoureusement isomorphe, à savoir autant concernant le sens lexical que le sens compositionnel. Si des ajustements sont nécessaires via une forme d'approximation, encore doit-on en régler la cohérence. Ceci éviterait p. ex. de traduire mal de cour et mal de mer par la même séquence, ou encore de maintenir une approximation préjudiciable à la compréhension de $\mathrm{L} 1$ et à son usage (armée, poignée, voler, gérer sont également des lexèmes du français, qui n'entretiennent pas une relation de synonymie stricte avec les SF qu'ils sont censés définir; les conditions d'emploi respectives des items doivent donc être exposées).

57. La traduction littérale est fournie entre crochets quand elle diverge de la signification de la séquence L1.

58. Qui reste souvent du domaine de l'horizon tant la réalité lexicale peut se montrer parfois retorse à toute catégorisation.

59. Sous-entendu pour nous qu'il n'aura pas à s'exprimer en L1, c'est-à-dire à produire des énoncés qu'il aura lui-même construits.

60. $[\mathrm{K}]$ et $[\mathrm{R}]$ ne se sont pas recopiés mutuellement. Ils ont éventuellement consulté un monolingue français ou un bilingue français-X, $\mathrm{X}$-français.

\section{RÉFÉRENCES}

Benveniste, E. (1974a) : «Fondements syntaxiques de la composition nominale», Problèmes de linguistique générale, T.2, Paris, Gallimard.

Benveniste, E. (1974b) : «Formes nouvelles de la composition nominale», Problèmes de linguistique générale, T.2, Paris, Gallimard.

Binon, J., Verlinde, S., Selva, T. et G. Petit : Dictionnaire d'apprentissage du français langue étrangère et langue seconde (DAFLES), <www.kuleuven.ac.be/dafles $>$.

Binon, J., Verlinde, S., Van Dyck, J. et A. Bertels (2000): Dictionnaire d'apprentissage du français des affaires (DAFA), Paris, Didier, <www.projetdafa.net/>.

CAdiot, P. et F. Nemo (1997c) : «Propriétés extrinsèques en sémantique lexicale», French Language Studies 7, Cambridge University Press, pp. 127-146.

Cadiot, P. et F. Lebas (dir.) (2003): «La constitution extrinsèque du référent», Langages 150, Paris, Larousse.

CAdiot, P. (1997a): «Aux sources de la polysémie lexicale», Langue française 113, pp. 3-11.

Cadiot, P. et F. Nemo (1997b) : «Pour une sémiogenèse du nom », Langue française 113, pp. 2434.

Cadiot, P. et F. Nemo (1997d): «Analytique des doubles caractérisations», Sémiotiques 13, pp. 123-143.

Cadiot, P. et L. Tracy (1997e): «On n’a pas toujours sa tête sur les épaules», Sémiotiques 13, pp. 105-121.

Camus, R. et S. De Vogüé (dir.) (2004): «Variation sémantique et syntaxique des unités lexicales: étude de six verbes français», Linx 50, Université Paris X, Nanterre.

Corbin, D. et M. Temple (1994) : «Le monde des mots et des sens construits: catégories sémantiques, catégories référentielles», Cahiers de lexicologie 65, pp. 5-28.

Corbin, D. (1987): Morphologie dérivationnelle et structuration du lexique, Niemeyer, Tübingen, Presses du Septentrion, Université de Lille.

FRADIN, B. (2003) : Nouvelles approches en morphologie, Paris, PUF.

Franckel, J.-J. et D. Lebaud (1990): Les figures du sujet, Paris, Ophrys.

Franckel, J.-J., Paillard, D. et E. Saunier (1996): «Modes de régulation de la variation sémantique d'une unité lexicale: le cas du verbe passer», in Fiala, P., Lafon, P. et M.-F. Piguet (dir.), La locution: entre syntaxe et pragmatique, Klincksieck, Paris.

Gross, G. (1988): «Degré de figement des noms composés», Langages 90, pp. 57-72.

Gross, G. (1996): Les expressions figées en français, Paris, Ophrys.

Grossman, F. et A. Tutin (dir.) (2002): «Lexique: recherches actuelles», Revue française de linguistique appliquée 7-1, Amsterdam-Paris, De Werelt-Université Paris VII.

Kleiber, G. (1984) : «Dénomination et relations dénominatives », Langages 76, pp. 77-94. 
Mathieu-Colas, M. (1996): "Typologie de la composition nominale», Cahiers de lexicologie 69, pp. 65-118.

Mejri, S. (1997): Le figement lexical, Tunis, Publications de la Faculté des Lettres de la Manouba.

Mejri, S., Gross, G., Clas, A. et T. Baccouche (dir.) (1998): Le figement lexical, Actes des Premières Rencontres Linguistiques Méditerranéennes, Tunis, CERES.

MejRI, S. (2000) : «Figement et dénomination», Meta 45-4, pp. 609-621.

Mejri, S., Baccouche, T., Clas, A. et G. Gross (dir.) (2000a): La traduction, théories et pratiques, actes du colloque international Traduction humaine, traduction automatique, interprétation (Tunis 28, 29, 30 septembre 2000), Tunis, Publications de l'ENS.

Mejri, S., Baccouche, T., Clas, A. et G. Gross (dir.) (2000b): La traduction: diversité linguistique et pratiques courantes, actes du colloque international Traduction humaine, traduction automatique, interprétation, (Tunis 28, 29, 30 septembre 2000), Tunis, Cahiers du C.E.R.E.S., Série Linguistique 11.

Mejri, S., Baccouche, T., Clas, A. et G. Gross (dir.) (2003): Traduire la langue, Traduire la culture, Tunis-Paris, Maisonneuve et Larose, Sud éditions.

Mejri, S. (dir.) (2004) : L'espace euro-méditerranéen: Une idiomaticité partagée, Actes du colloque international (Hammamet 19, 20, 21 septembre 2003), Tome 2, Tunis, Cahiers du C.E.R.E.S., Série Linguistique 12.

Petit, G. (1998): «Remarques sur la structuration sémiotique des locutions familières», in Mejri, S., Gross, G., Clas, A. et T. Baccouche (dir.), Le figement lexical, Actes des Premières Rencontres Linguistiques Méditerranéennes, Tunis, CERES.

Petit, G. (2003): "Lemmatisation et figement lexical: les locutions de type SV", Cahiers de lexicologie 82, pp. 127-158.

Petit, G. (2004): «L'idiomaticité de l'autre: propositions pour un dictionnaire bilingue d'apprentissage français-grec», in MEJRI, S. (dir.), L'espace euro-méditerranéen: Une idiomaticité partagée, Actes du colloque international (Hammamet 19, 20, 21 septembre 2003), Tome 2, Tunis, Cahiers du C.E.R.E.S., Série Linguistique 12.

Rey, A. (1970): La lexicologie, Paris, Klincksieck.

Rey, A. (1976): Théories du signe et du sens, Paris, Klincksieck.

Szende, T. (dir.) (2003): Les écarts culturels dans les dictionnaires bilingues, Paris, Honoré Champion.

Temple, M. (1996): Pour une sémantique des mots construits, Lille, Presses universitaires du Septentrion.

\section{Dictionnaires}

$\Lambda \varepsilon \xi ı \kappa o ́ ~ \tau \eta \varsigma ~ \kappa o เ v \eta ́ \varsigma ~ v \varepsilon o \varepsilon \lambda \lambda \eta v \iota \kappa \eta ́ \varsigma$, Institut d'études néohelléniques, Université Aristote de Thessalonique, 1998.

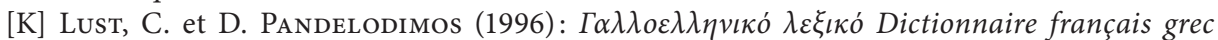
moderne, Athènes, Librairie Kauffmann.

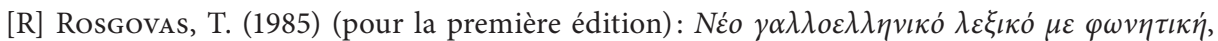
Athènes, Rosgovas. 